\title{
Existence and Asymptotic Behavior of Traveling Wave Fronts for a Time-Delayed Degenerate Diffusion Equation
}

\author{
Weifang Yan and Rui Liu \\ Department of Mathematics, South China University of Technology, Guangzhou 510640, China \\ Correspondence should be addressed to Rui Liu; scliurui@scut.edu.cn
}

Received 15 November 2012; Revised 15 February 2013; Accepted 18 February 2013

Academic Editor: Peixuan Weng

Copyright ( 2013 W. Yan and R. Liu. This is an open access article distributed under the Creative Commons Attribution License, which permits unrestricted use, distribution, and reproduction in any medium, provided the original work is properly cited.

\begin{abstract}
This paper is concerned with traveling wave fronts for a degenerate diffusion equation with time delay. We first establish the necessary and sufficient conditions to the existence of monotone increasing and decreasing traveling wave fronts, respectively. Moreover, special attention is paid to the asymptotic behavior of traveling wave fronts connecting two uniform steady states. Some previous results are extended.
\end{abstract}

\section{Introduction}

In this paper, we consider the traveling wave fronts for the following reaction diffusion equation with Hodgkin-Huxley source:

$$
\frac{\partial u}{\partial t}=\frac{\partial^{2} u^{m}}{\partial x^{2}}+f\left(u, u_{\tau}\right), \quad t \geq 0, x \in \mathbb{R}
$$

where $m>0, f(u, v)=u^{p}(1-u)^{q}(v-a), p>0, q>0$, $m+p>1, a \in(0,1)$ is a constant, and $u_{\tau}(x, t)=u(x, t-\tau)$ for $\tau>0$.

In 1952, Hodgkin and Huxley [1] proposed the HodgkinHuxley (H-H) equation

$$
\frac{d u}{d t}=u(t)(1-u(t))(u(t-\tau)-a), \quad a \in(0,1),
$$

which describes the propagation of a voltage pulse through the nerve axon of a squid. Recently, more and more attention has been paid to the linear and semilinear parabolic equations with and without time delay; see, for example, [2-7]. A natural extension of the $\mathrm{H}-\mathrm{H}$ model is the following linear diffusion equation:

$$
\frac{\partial u}{\partial t}=\frac{\partial^{2} u}{\partial x^{2}}+u(1-u)\left(u_{\tau}-a\right) .
$$

For this equation, there have been many interesting results on the existence and stability of the traveling wave solutions, for instance, [8-10]. By a traveling wave solution, we mean a solution $u(x, t)$ of $(3)$ of the form $u(x, t)=\varphi(x+c t)$ with the wave speed $c$.

On the other hand, the classical research of traveling waves for the standard linear diffusion equations with various sources has been extended to some degenerate or singular diffusion equations. For example, Aronson [11] considered the following equation:

$$
\frac{\partial u}{\partial t}=\frac{\partial^{2} u^{m}}{\partial x^{2}}+u(1-u)(u-a), \quad a \in(0,1) .
$$

When $m>1$, the equation degenerates at $u=0$. Hence, it has a different feature from the case $m=1$; that is, if the initial distribution of $u(x, t)$ has compact support, then $u(x, t)$ also has compact support for each $t>0$. When $m>1, a \in(0$, $1 / 2$ ), Aronson [11] showed that (4) possesses a unique sharp traveling wave solution with positive wave speed. Hosono [12] solved the existence problem of traveling wave solutions for (4) especially with nonpositive wave speed and discussed the shape of the solutions. Sánchez-Garduño and Maini [13] considered the following degenerate diffusion equation:

$$
\begin{array}{r}
\frac{\partial u}{\partial t}=\frac{\partial}{\partial x}\left(D(u) \frac{\partial u}{\partial x}\right)+u(1-u)(u-a), \\
a \in(0,1), D(0)=0,
\end{array}
$$

and obtained the existence of traveling wave solutions of smooth or sharp (oscillatory and monotone) type. 
For other papers concerning the traveling wave solutions for degenerate diffusion equations without time delay, see [14-22]. From these results, we see that an obvious difference between the linear diffusion equations and the degenerate diffusion equations is that, in the degenerate diffusion case, there may exist traveling wave fronts of sharp type; that is, the support of the solution is bounded above or below, and at the boundary of the support, the derivative of the traveling wave solution is discontinuous. However, in the linear diffusion case, all traveling wave fronts are of smooth type; that is, the solutions are classical solutions, which approach the steady states at infinity.

As far as we know, there are only two articles dealing with the traveling wave solutions for degenerate diffusion equations with time delay. In $[23,24]$, Jin et al. considered the following time-delayed Newtonian filtration and non-Newtonian filtration equations:

$$
\begin{gathered}
\frac{\partial u}{\partial t}=\frac{\partial^{2} u^{m}}{\partial x^{2}}+u(1-u)\left(u_{\tau}-a\right) \\
\frac{\partial u}{\partial t}=\frac{\partial}{\partial x}\left(\left|\frac{\partial u}{\partial x}\right|^{p-2} \frac{\partial u}{\partial x}\right)+u^{q}(1-u)\left(u_{\tau}-a\right) .
\end{gathered}
$$

By using the shooting method together with the comparison technique, they first obtained the necessary and sufficient conditions to the existence of monotone increasing and decreasing traveling wave solutions, respectively, and then gave an accurate estimation on the convergent rate for the semifinite or infinite traveling waves.

Motivated by [23, 24], in this paper, we discuss the existence and asymptotic behavior of traveling wave fronts for (1). Let $u(x, t)=\varphi(\xi)$ with $\xi=x+c t$. Then, (1) is transformed into the following form:

$$
c \varphi^{\prime}(\xi)=\left(\varphi^{m}\right)^{\prime \prime}(\xi)+\varphi(\xi)^{p}(1-\varphi(\xi))^{q}\left(\varphi_{c \tau}(\xi)-a\right),
$$

where $\varphi_{c \tau}(\xi)=\varphi(\xi-c \tau)$.

Before going further, we first give the definition of sharpand smooth-type traveling wave fronts.

Definition 1. A function $\varphi(\xi)$ is called a traveling wave front with wave speed $c>0$ if there exist $\xi_{a}, \xi_{b}$ with $-\infty \leq \xi_{a}<$ $\xi_{b} \leq+\infty$ such that $\varphi \in C^{2}\left(\xi_{a}, \xi_{b}\right)$ is monotonic increasing and

$$
\begin{gathered}
c \varphi^{\prime}(\xi)=\left(\varphi^{m}\right)^{\prime \prime}(\xi)+\varphi(\xi)^{p}(1-\varphi(\xi))^{q}\left(\varphi_{c \mathcal{c}}(\xi)-a\right), \\
\xi \in\left(\xi_{a}, \xi_{b}\right), \\
\varphi(\xi)=0 \quad \text { for } \xi_{a}-c \tau<\xi \leq \xi_{a}, \\
\varphi\left(\xi_{b}\right)=1, \\
\left(\varphi^{m}\right)^{\prime}\left(\xi_{a}\right)=\left(\varphi^{m}\right)^{\prime}\left(\xi_{b}\right)=0,
\end{gathered}
$$

or there exist $\tilde{\xi}_{a}, \tilde{\xi}_{b}$ with $-\infty \leq \tilde{\xi}_{a}<\tilde{\xi}_{b} \leq+\infty$ such that $\varphi \in C^{2}\left(\widetilde{\xi}_{a}, \widetilde{\xi}_{b}\right)$ is monotonic decreasing and

$$
\begin{gathered}
c \varphi^{\prime}(\xi)=\left(\varphi^{m}\right)^{\prime \prime}(\xi)+\varphi(\xi)^{p}(1-\varphi(\xi))^{q}\left(\varphi_{c \tau}(\xi)-a\right), \\
\xi \in\left(\widetilde{\xi}_{a}, \widetilde{\xi}_{b}\right), \\
\varphi(\xi)=1 \quad \text { for } \widetilde{\xi}_{a}-c \tau<\xi \leq \widetilde{\xi}_{a}, \\
\varphi\left(\widetilde{\xi}_{b}\right)=0, \\
\left(\varphi^{m}\right)^{\prime}\left(\widetilde{\xi}_{a}\right)=\left(\varphi^{m}\right)^{\prime}\left(\widetilde{\xi}_{b}\right)=0 .
\end{gathered}
$$

(i) If $\xi_{a}>-\infty$ and $\varphi^{\prime}\left(\xi_{a}^{+}\right) \neq 0$, then $\varphi(\xi)$ is called an increasing sharp-type traveling wave front (see Figure 1(a)).

(ii) If $\xi_{a}=-\infty$ or $\varphi^{\prime}\left(\xi_{a}^{+}\right)=0$, then $\varphi(\xi)$ is called an increasing smooth-type traveling wave front (see Figure 2(a)).

Similarly, we have the following.

(iii) If $\tilde{\xi}_{b}<+\infty$ and $\varphi^{\prime}\left(\widetilde{\xi}_{b}^{-}\right) \neq 0$, then $\varphi(\xi)$ is called a decreasing sharp-type traveling wave front (see Figure 1(b)).

(iv) If $\widetilde{\xi}_{b}=+\infty$ or $\varphi^{\prime}\left(\widetilde{\xi}_{b}^{-}\right)=0$, then $\varphi(\xi)$ is called a decreasing smooth-type traveling wave front (see Figure 2(b)).

$$
\begin{array}{r}
\text { Let } \psi(\xi)=\left(\varphi^{m}\right)^{\prime}(\xi) \text {. Then, (8) or }(10) \text { is transformed into } \\
\varphi^{\prime}(\xi)=\frac{1}{m} \varphi^{1-m}(\xi) \psi(\xi), \\
\psi^{\prime}(\xi)=\frac{c}{m} \varphi^{1-m}(\xi) \psi(\xi)-\varphi(\xi)^{p}(1-\varphi(\xi))^{q}\left(\varphi_{\mathcal{~} \tau}(\xi)-a\right) .
\end{array}
$$

Furthermore, by (9) or (11), we give the asymptotic boundary conditions for traveling wave fronts as follows:

$$
\begin{gathered}
\varphi(\xi)=0 \quad \text { for } \xi_{a}-c \tau<\xi \leq \xi_{a}, \\
\varphi\left(\xi_{b}\right)=1, \\
\psi\left(\xi_{a}\right)=\psi\left(\xi_{b}\right)=0
\end{gathered}
$$

or

$$
\begin{gathered}
\varphi(\xi)=1 \quad \text { for } \widetilde{\xi}_{a}-c \tau<\xi \leq \widetilde{\xi}_{a}, \\
\varphi\left(\widetilde{\xi}_{b}\right)=0, \\
\psi\left(\widetilde{\xi}_{a}\right)=\psi\left(\widetilde{\xi}_{b}\right)=0 .
\end{gathered}
$$

If $\psi(\xi)$ is strictly positive or negative for $0<\varphi<1$, then (12) is equivalent to

$$
\frac{d \psi}{d \varphi}=c-\frac{m \varphi^{m+p-1}(1-\varphi)^{q}\left(\varphi_{c \tau}-a\right)}{\psi} .
$$




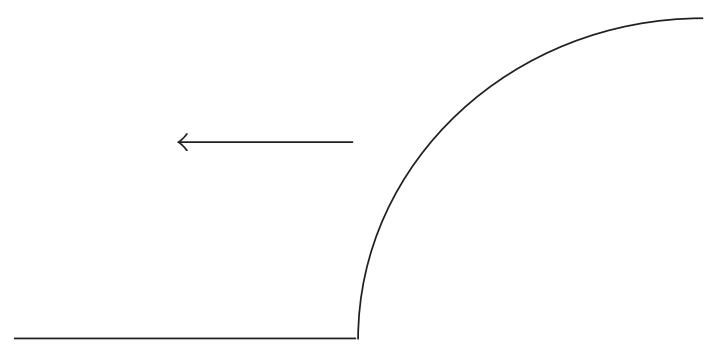

(a)

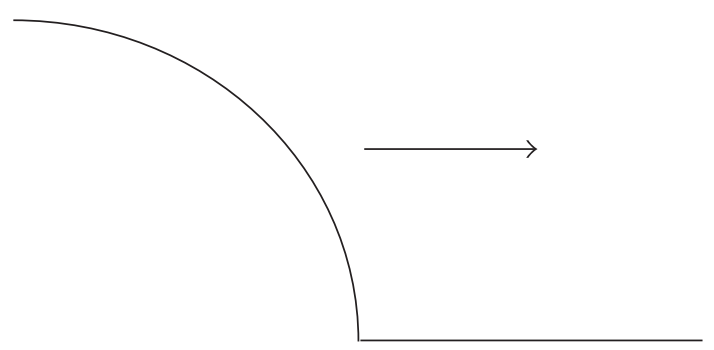

(b)

FIgURE 1: Sharp-type traveling wave fronts. (a) Monotonic increasing. (b) Monotonic decreasing.

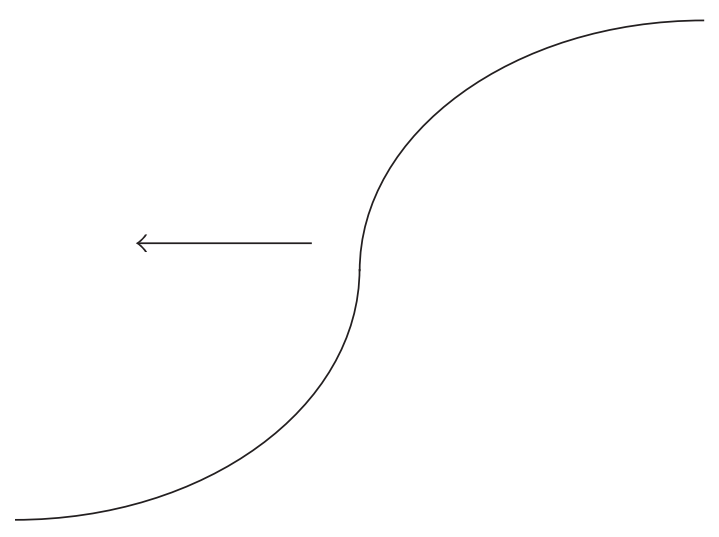

(a)

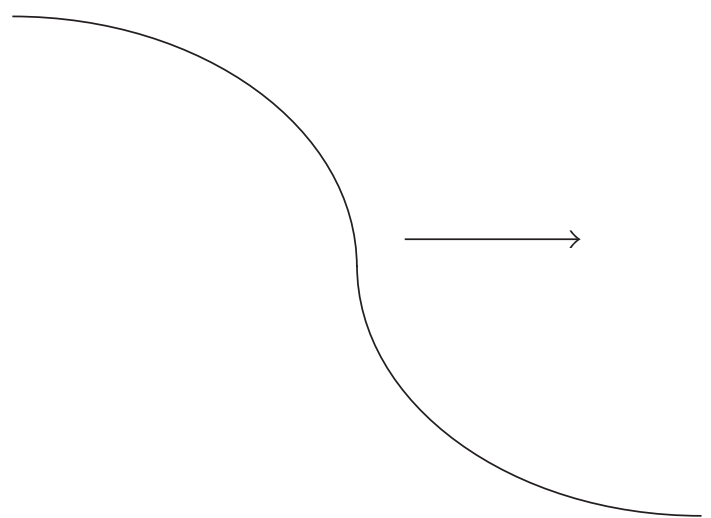

(b)

FIGURE 2: Smooth-type traveling wave fronts. (a) Monotonic increasing. (b) Monotonic decreasing.

Clearly, for any given $\varphi>0$, if

$$
\int_{0}^{\varphi} \frac{m \sigma^{m-1}}{\psi(\sigma)} d \sigma=+\infty
$$

then $\varphi_{c \tau}$ can be defined by

$$
c \mathcal{c}=\int_{\varphi_{c \tau}}^{\varphi} \frac{m \sigma^{m-1}}{\psi(\sigma)} d \sigma .
$$

However, if for some $\varphi>0$,

$$
\int_{0}^{\varphi} \frac{m \sigma^{m-1}}{\psi(\sigma)} d \sigma<+\infty
$$

then $\int_{0}^{\varphi}\left(m \sigma^{m-1} / \psi(\sigma)\right) d \sigma$ may be less than $c \tau$ when $\varphi$ is near 0 . Therefore, the previous definition is not reasonable. In what follows, we give the definition of $\varphi_{c \tau}$.

(i) If $\psi$ is positive, define $\varphi_{c \tau}$ by

$$
\begin{gathered}
c \tau=\int_{\varphi_{c \tau}}^{\varphi} \frac{m \sigma^{m-1}}{\psi(\sigma)} d \sigma, \quad \text { if } \int_{0}^{\varphi} \frac{m \sigma^{m-1}}{\psi_{+}(\sigma)} d \sigma \geq c \tau, \\
\varphi_{c \tau}=0, \quad \text { if } \int_{0}^{\varphi} \frac{m \sigma^{m-1}}{\psi_{+}(\sigma)} d \sigma<c \tau,
\end{gathered}
$$

where $\psi_{+}$is a solution of the following problem:

$$
\begin{gathered}
\frac{d \psi}{d \varphi}=c+\frac{m a \varphi^{m+p-1}(1-\varphi)^{q}}{\psi}, \quad \text { for } \varphi \in(0,1), \\
\psi\left(0^{+}\right)=0, \\
\psi(\varphi)>0, \quad \text { for } \varphi \in(0,1) .
\end{gathered}
$$

(ii) If $\psi$ is negative, define $\varphi_{c \tau}$ by

$$
\begin{gathered}
c \tau=\int_{\varphi}^{\varphi_{c \tau}} \frac{m \sigma^{m-1}}{-\psi(\sigma)} d \sigma, \quad \text { if } \int_{\varphi}^{1} \frac{m \sigma^{m-1}}{-\psi_{-}(\sigma)} d \sigma \geq c \tau \\
\varphi_{c \tau}=1, \quad \text { if } \int_{\varphi}^{1} \frac{m \sigma^{m-1}}{-\psi_{-}(\sigma)} d \sigma<c \tau
\end{gathered}
$$

where $\psi_{-}$is a solution of the following problem:

$$
\begin{gathered}
\frac{d \psi}{d \varphi}=c-\frac{m(1-a) \varphi^{m+p^{-1}}(1-\varphi)^{q}}{\psi}, \text { for } \varphi \in(0,1), \\
\psi\left(1^{-}\right)=0, \\
\psi(\varphi)<0, \quad \text { for } \varphi \in(0,1) .
\end{gathered}
$$


Consider the following problem:

$$
\begin{gathered}
\frac{d \psi}{d \varphi}=c-\frac{m \varphi^{m+p-1}(1-\varphi)^{q}\left(\varphi_{c \tau}-a\right)}{\psi}, \\
\psi\left(0^{+}\right)=0, \quad \psi\left(1^{-}\right)=0 .
\end{gathered}
$$

In Sections 2 and 3, we will verify the following two conclusions are equivalent, that is, (1) $\varphi$ is a monotonic solution of the problem (8)-(9) (or (10)-(11)); (2) $\psi(\varphi)>0($ or $\psi(\varphi)<0)$ is a solution of the problem (23).

\section{Existence of Increasing Traveling Waves}

In this section, we aim to find a solution $\psi(\varphi)$ of the problem (23) with $\psi(\varphi)>0$ for $\varphi \in(0,1)$.

Since $\psi>0$, we see that $\varphi(\xi)$ is increasing in $\xi$, and so $\varphi_{c \tau}(\xi) \leq \varphi(\xi)$. Thus, to investigate the behavior of the trajectories $\psi_{c}(\varphi)$ of (23), we have to study the trajectories starting from $(0,0)$, since the property of $\psi_{c}$ at $\varphi$ depends on the behavior of $\psi_{c}$ at $\varphi_{c \tau}$ closely. Consider the following problem:

$$
\begin{gathered}
\frac{d \psi}{d \varphi}=c-\frac{m \varphi^{m+p-1}(1-\varphi)^{q}\left(\varphi_{c \tau}-a\right)}{\psi} \text { for } \varphi \in(0, \beta), \\
\psi\left(0^{+}\right)=0, \\
\psi(\varphi)>0 \text { for } \varphi \in(0, \beta),
\end{gathered}
$$

where $(0, \beta)$ with $\beta \leq 1$ is the maximal existence interval of the solution $\psi(\varphi)>0$. By $(24)$,

$$
\begin{aligned}
\frac{1}{2} \psi^{2}(\varphi) & =\int_{0}^{\varphi} c \psi(s) d s-m \int_{0}^{\varphi} s^{m+p-1}(1-s)^{q}\left(s_{c \tau}-a\right) d s \\
& \geq \int_{0}^{\varphi} c \psi(s) d s-m \int_{0}^{\varphi} s^{m+p-1}(1-s)^{q}(s-a) d s .
\end{aligned}
$$

This excludes $\psi\left(1^{-}\right)=0$ if $\int_{0}^{1} s^{m+p-1}(1-s)^{q}(s-a) d s \leq 0$. Thus, we only need to find the increasing traveling wave fronts for the case $\int_{0}^{1} s^{m+p-1}(1-s)^{q}(s-a) d s>0$. We first prove the following two lemmas.

Lemma 2. Assume that $0<\varphi_{0}<1$ and $\psi_{1}(\varphi), \psi_{2}(\varphi)$ are solutions of (24) corresponding to different wave speeds $c_{1}, c_{2}$, respectively, where $\psi_{1}\left(\varphi_{0}\right) \geq \psi_{2}\left(\varphi_{0}\right)>0, \psi_{1}(\varphi)>\psi_{2}(\varphi)>0$ for $0<\varphi<\varphi_{0}$, and $\varphi_{1}\left(\xi_{1}\right)=\varphi_{2}\left(\xi_{2}\right)=\varphi_{0}$. Then, $\varphi_{1}\left(\xi_{1}-s\right)<$ $\varphi_{2}\left(\xi_{2}-s\right)$ if $0<s<\int_{0}^{\varphi_{0}}\left(m \sigma^{m-1} / \psi_{2}(\sigma)\right) d \sigma$ and $\varphi_{1}\left(\xi_{1}-s\right)=$ $\varphi_{2}\left(\xi_{2}-s\right)=0$ if $s \geq \int_{0}^{\varphi_{0}}\left(m \sigma^{m-1} / \psi_{2}(\sigma)\right) d \sigma$.

Proof. Recalling (19), we see that

$$
\begin{gathered}
s=\int_{\varphi_{i s}}^{\varphi_{0}} \frac{m \sigma^{m-1}}{\psi_{i}(\sigma)} d \sigma \quad \text { if } \int_{0}^{\varphi_{0}} \frac{m \sigma^{m-1}}{\psi_{i}(\sigma)} d \sigma>s, \\
\varphi_{i s}=0 \text { if } \int_{0}^{\varphi_{0}} \frac{m \sigma^{m-1}}{\psi_{i}(\sigma)} d \sigma \leq s,
\end{gathered}
$$

where $\varphi_{i s}=\varphi_{i}\left(\xi_{i}-s\right), i=1,2$. The desired conclusion follows immediately.

Lemma 3. For any given $\tau>0$, and $c_{1}>c_{2} \geq 0$, let $\psi_{1}(\varphi)$, $\psi_{2}(\varphi)$ be solutions of (24) corresponding to $c_{1}, c_{2}$, respectively. Then, $\psi_{1}(\varphi)>\psi_{2}(\varphi)$ for any $\varphi \in\left(0, \beta_{2}\right)$, where $\left(0, \beta_{2}\right)$ is the maximal existence interval of the solution $\psi_{2}(\varphi)>0$. In addition, $\psi_{1}\left(\beta_{2}\right)>0$. (See Figure 3(a).)

Proof. We first show that $\psi_{1}(\varphi)>\psi_{2}(\varphi)$ for sufficiently small $\varphi>0$. The argument consists of three cases, $m+p>2, m+$ $p=2$, and $1<m+p<2$. have

(i) Consider the case $m+p>2$. According to (24), we

$$
\frac{d \psi}{d \varphi}=c+\frac{m \varphi^{m+p-1}(1-\varphi)^{q}\left(a-\varphi_{c \tau}\right)}{\psi} .
$$

Noticing that $\varphi_{\mathcal{c} \tau} \leq \varphi$, we have for $\varphi \leq a$ that

$$
\frac{d \psi}{d \varphi} \geq c .
$$

Integrating from 0 to $\varphi$ yields

$$
\psi(\varphi) \geq c \varphi .
$$

We further have

$$
\begin{aligned}
\frac{d \psi}{d \varphi} & =c+\frac{m \varphi^{m+p-1}(1-\varphi)^{q}\left(a-\varphi_{c \tau}\right)}{\psi} \\
& \leq c+\frac{m \varphi^{m+p-1}(1-\varphi)^{q}\left(a-\varphi_{c \tau}\right)}{c \varphi} \\
& \leq c+\frac{a m}{c} \varphi^{m+p-2} .
\end{aligned}
$$

Integrating from 0 to $\varphi$ gives

$$
\psi(\varphi) \leq c \varphi+\frac{a m}{c(m+p-1)} \varphi^{m+p-1} .
$$

That is,

$$
\psi(\varphi) \sim c \varphi \text { as } \varphi \longrightarrow 0^{+} .
$$

Therefore, $\psi_{1}(\varphi)>\psi_{2}(\varphi)$ for sufficiently small $\varphi>0$.

(ii) Consider the case $m+p=2$. Similar to (i), we have

$$
\psi(\varphi) \geq c \varphi,
$$

and, hence,

$$
\psi(\varphi) \leq\left(c+\frac{a m}{c}\right) \varphi .
$$

Consider the sequences $\left\{A_{n}\right\}$ and $\left\{B_{n}\right\}$, where

$$
\begin{gathered}
A_{1}=c, \quad B_{1}=c+\frac{a m}{c}, \\
A_{n}=c+\frac{(1-\varepsilon)^{q}(a-\varepsilon) m}{B_{n-1}}, \\
B_{n}=c+\frac{a m}{A_{n-1}}, \quad n=2,3, \ldots,
\end{gathered}
$$




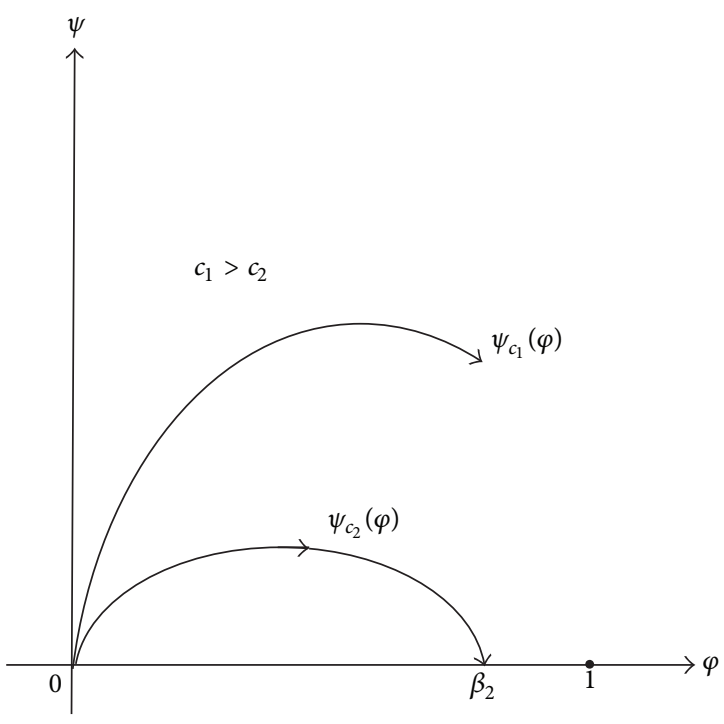

(a)

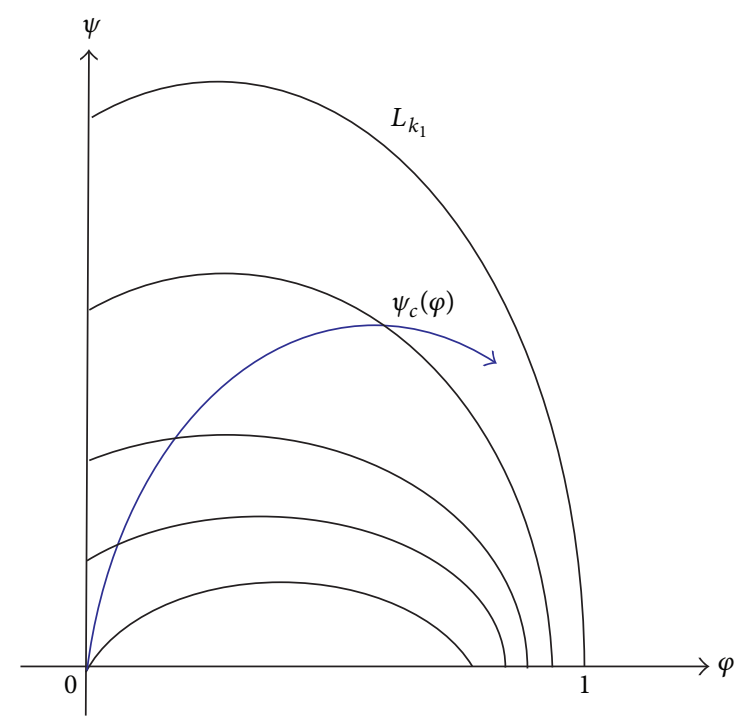

(b)

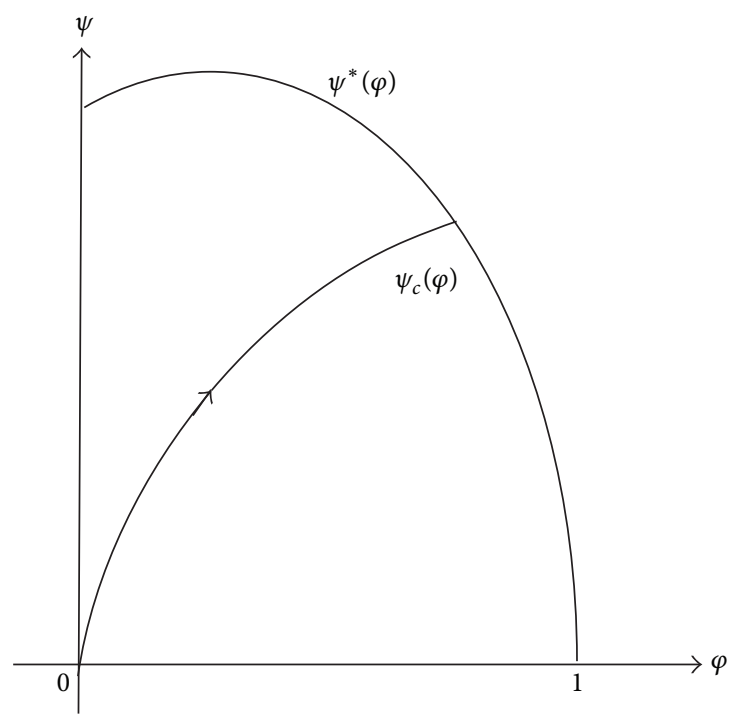

(c)

FiguRE 3: The properties of the trajectory $\psi_{c}(\varphi)$. (a) The monotonicity of $\psi_{c}(\varphi)$ on $c$. (b) The trajectory $\psi_{c}(\varphi)$ wanders through $L_{k}$. (c) The trajectory $\psi_{c}(\varphi)$ intersects with $\psi^{*}(\varphi)$ for large $c$.

and $\varepsilon>0$ is sufficiently small. Noticing that $A_{1}<B_{1}$ and $A_{1}<A_{2}$, by induction, we obtain

$$
\begin{aligned}
A_{1} & <A_{2}=A_{3}<A_{4}=A_{5}<\cdots<A_{n} \\
& <\cdots<B_{n}<\cdots<B_{4}=B_{3}<B_{2}=B_{1} .
\end{aligned}
$$

For $0<\varphi<\varepsilon$, if

$$
A_{n-1} \varphi \leq \psi(\varphi) \leq B_{n-1} \varphi,
$$

then

$$
\begin{aligned}
\frac{d \psi}{d \varphi} & =c+\frac{m \varphi(1-\varphi)^{q}\left(a-\varphi_{c \tau}\right)}{\psi} \\
& \leq c+\frac{m \varphi(1-\varphi)^{q}\left(a-\varphi_{c \tau}\right)}{A_{n-1} \varphi}
\end{aligned}
$$

$$
\begin{aligned}
& \leq c+\frac{a m}{A_{n-1}}, \\
\frac{d \psi}{d \varphi} & =c+\frac{m \varphi(1-\varphi)^{q}\left(a-\varphi_{c \tau}\right)}{\psi} \\
& \geq c+\frac{m \varphi(1-\varphi)^{q}\left(a-\varphi_{c \tau}\right)}{B_{n-1} \varphi} \\
& \geq c+\frac{(1-\varepsilon)^{q}(a-\varepsilon) m}{B_{n-1}} .
\end{aligned}
$$

Integrating from 0 to $\varphi$ yields

$$
A_{n} \varphi \leq \psi(\varphi) \leq B_{n} \varphi .
$$


Thus, we have

$$
\begin{gathered}
\lim _{n \rightarrow \infty} A_{n}=A^{*}(\varepsilon), \quad \lim _{n \rightarrow \infty} B_{n}=B^{*}(\varepsilon), \\
A^{*}(\varepsilon) \varphi \leq \psi(\varphi) \leq B^{*}(\varepsilon) \varphi,
\end{gathered}
$$

with $A^{*}(\varepsilon)$ and $B^{*}(\varepsilon)$ satisfying

$$
A^{*}(\varepsilon)=c+\frac{(1-\varepsilon)^{q}(a-\varepsilon) m}{B^{*}(\varepsilon)}, \quad B^{*}(\varepsilon)=c+\frac{a m}{A^{*}(\varepsilon)} .
$$

Letting $\varepsilon \rightarrow 0$, we obtain

$$
\lim _{\varepsilon \rightarrow 0} A^{*}(\varepsilon)=\lim _{\varepsilon \rightarrow 0} B^{*}(\varepsilon)=\frac{\sqrt{c^{2}+4 m a}+c}{2} .
$$

That is,

$$
\psi(\varphi) \sim \frac{\sqrt{c^{2}+4 m a}+c}{2} \varphi \text { as } \varphi \longrightarrow 0^{+} .
$$

Therefore, $\psi_{1}(\varphi)>\psi_{2}(\varphi)$ for sufficiently small $\varphi>0$.

(iii) Consider the case $1<m+p<2$. Notice that

$$
\frac{d \psi}{d \varphi} \geq \frac{m \varphi^{m+p-1}(1-\varphi)^{q}(a-\varphi)}{\psi},
$$

which means that

$$
\begin{aligned}
\psi^{2}(\varphi) & \geq \int_{0}^{\varphi} 2 m s^{m+p-1}(1-s)^{q}(a-s) d s \\
& =\frac{2 m a}{m+p} \varphi^{m+p}+o\left(\varphi^{m+p}\right) ;
\end{aligned}
$$

that is,

$$
\psi(\varphi) \geq \sqrt{\frac{2 m a}{m+p}} \varphi^{(m+p) / 2}+o\left(\varphi^{(m+p) / 2}\right) .
$$

Consequently,

$$
\begin{aligned}
\frac{d \psi}{d \varphi} & \leq c+\frac{m a \varphi^{m+p-1}}{\psi} \\
& \leq c+\frac{m a \varphi^{m+p-1}}{\sqrt{2 m a /(m+p)} \varphi^{(m+p) / 2}+o\left(\varphi^{(m+p) / 2}\right)} \\
& =c+\sqrt{\frac{m a(m+p)}{2}} \varphi^{(m+p-2) / 2}+o\left(\varphi^{(m+p-2) / 2}\right) .
\end{aligned}
$$

That is,

$$
\psi(\varphi) \leq c \varphi+\sqrt{\frac{2 m a}{m+p}} \varphi^{(m+p) / 2}+o\left(\varphi^{(m+p) / 2}\right) .
$$

Thus, we have

$$
\psi(\varphi)=\sqrt{\frac{2 m a}{m+p}} \varphi^{(m+p) / 2}+o\left(\varphi^{(m+p) / 2}\right) .
$$

Recalling (46), we see that

$$
\begin{aligned}
\frac{d \psi^{2}}{d \varphi}= & 2 c \psi+2 m \varphi^{m+p-1}(1-\varphi)^{q}\left(a-\varphi_{c \tau}\right) \\
\geq & 2 c \sqrt{\frac{2 m a}{m+p}} \varphi^{(m+p) / 2}+2 m \varphi^{m+p-1}(1-\varphi)^{q}(a-\varphi) \\
& +o\left(\varphi^{(m+p) / 2}\right),
\end{aligned}
$$

which implies that

$$
\begin{aligned}
\psi^{2}(\varphi) \geq & \frac{4 c}{m+p+2} \sqrt{\frac{2 m a}{m+p}} \varphi^{(m+p+2) / 2} \\
& +\frac{2 m a}{m+p} \varphi^{m+p}+o\left(\varphi^{(m+p+2) / 2}\right) .
\end{aligned}
$$

On the other hand, by (49), we have

$$
\begin{aligned}
\frac{d \psi^{2}}{d \varphi} & \leq 2 c \psi+2 m a \varphi^{m+p-1} \\
& =2 c \sqrt{\frac{2 m a}{m+p}} \varphi^{(m+p) / 2}+2 m a \varphi^{m+p-1}+o\left(\varphi^{(m+p) / 2}\right),
\end{aligned}
$$

and, hence,

$$
\begin{aligned}
\psi^{2}(\varphi) \leq & \frac{4 c}{m+p+2} \sqrt{\frac{2 m a}{m+p}} \varphi^{(m+p+2) / 2} \\
& +\frac{2 m a}{m+p} \varphi^{m+p}+o\left(\varphi^{(m+p+2) / 2}\right) .
\end{aligned}
$$

Summing up, we arrive at

$$
\begin{aligned}
\psi^{2}(\varphi)= & \frac{4 c}{m+p+2} \sqrt{\frac{2 m a}{m+p}} \varphi^{(m+p+2) / 2}+\frac{2 m a}{m+p} \varphi^{m+p} \\
& +o\left(\varphi^{(m+p+2) / 2}\right), \quad \text { as } \varphi \longrightarrow 0^{+}
\end{aligned}
$$

which implies that $\psi_{1}(\varphi)>\psi_{2}(\varphi)$ for sufficiently small $\varphi>0$.

We claim that $\psi_{1}(\varphi)>\psi_{2}(\varphi)$ for any $\varphi \in\left(0, \beta_{2}\right)$. Suppose for contradiction that there exists $\varphi_{0} \in\left(0, \beta_{2}\right)$ such that $\psi_{1}\left(\varphi_{0}\right)=\psi_{2}\left(\varphi_{0}\right)=\psi_{0}$ and $\psi_{1}(\varphi)>\psi_{2}(\varphi)$ for $\varphi \in\left(0, \varphi_{0}\right)$. Then,

$$
\psi_{1}^{\prime}\left(\varphi_{0}\right) \leq \psi_{2}^{\prime}\left(\varphi_{0}\right)
$$

which means that

$$
\begin{aligned}
c_{1}+\frac{m \varphi_{0}^{m+p-1}\left(1-\varphi_{0}\right)^{q}\left(a-\varphi_{0 c_{1} \tau}\right)}{\psi_{0}} \\
\quad \leq c_{2}+\frac{m \varphi_{0}^{m+p-1}\left(1-\varphi_{0}\right)^{q}\left(a-\varphi_{0 c_{2} \tau}\right)}{\psi_{0}} ;
\end{aligned}
$$


that is,

$$
0<c_{1}-c_{2} \leq \frac{m \varphi_{0}^{m+p-1}\left(1-\varphi_{0}\right)^{q}}{\psi_{0}}\left(\varphi_{0 c_{1} \tau}-\varphi_{0 c_{2} \tau}\right) .
$$

Thus,

$$
\varphi_{0 c_{1} \tau}>\varphi_{0 c_{2} \tau}
$$

Denote that $\varphi_{1}\left(\xi_{1}\right)=\varphi_{2}\left(\xi_{2}\right)=\varphi_{0}$. By Lemma 2, we have

$$
\varphi_{1}\left(\xi_{1}-c_{1} \tau\right) \leq \varphi_{2}\left(\xi_{2}-c_{1} \tau\right) \leq \varphi_{2}\left(\xi_{2}-c_{2} \tau\right),
$$

which means that $\varphi_{0 c_{1} \tau} \leq \varphi_{0 c_{2} \tau}$, a contradiction.

In what follows, we will show that $\psi_{1}\left(\beta_{2}\right)>0$. Recalling the first equation of (24), we infer that

$$
\frac{1}{2} \frac{d \psi_{i}^{2}}{d \varphi}=c_{i} \psi_{i}-m \varphi^{m+p-1}(1-\varphi)^{q}\left(\varphi_{c_{i} \tau}-a\right), \quad i=1,2 .
$$

By Lemma 2, for any $\varphi_{1}\left(\xi_{1}\right)=\varphi_{2}\left(\xi_{2}\right)<\beta_{2}$, we have

$$
\varphi_{1}\left(\xi_{1}-c_{1} \tau\right) \leq \varphi_{2}\left(\xi_{2}-c_{1} \tau\right) \leq \varphi_{2}\left(\xi_{2}-c_{2} \tau\right),
$$

since $\psi_{1}>\psi_{2}$. Thus, we obtain

$$
\frac{d \psi_{1}^{2}}{d \varphi}>\frac{d \psi_{2}^{2}}{d \varphi}
$$

for any $\varphi \in\left(0, \beta_{2}\right)$. Integrating the previous inequality from $\varphi_{0}$ to $\varphi$ for any $0<\varphi_{0}<\varphi<\beta_{2}$ yields

$$
\psi_{1}^{2}(\varphi)>\psi_{2}^{2}(\varphi)+\psi_{1}^{2}\left(\varphi_{0}\right)-\psi_{2}^{2}\left(\varphi_{0}\right)
$$

Letting $\varphi \rightarrow \beta_{2}$ gives

$$
\psi_{1}^{2}\left(\beta_{2}\right) \geq \psi_{1}^{2}\left(\varphi_{0}\right)-\psi_{2}^{2}\left(\varphi_{0}\right)>0 ;
$$

namely, $\psi_{1}\left(\beta_{2}\right)>0$. The proof is completed.

To deal with the behavior of the trajectories $\psi_{c}(\varphi)$ of the problem (24), we introduce the level set

$L_{k}$

$$
=\left\{(\varphi, \psi) \in \mathbb{R}^{2} ; \frac{1}{2} \psi^{2}+m \int_{0}^{\varphi} s^{m+p-1}(1-s)^{q}(s-a) d s=k\right\}
$$

for any $k \geq 0$. Clearly, for $c=0$, the level sets $\left\{L_{k}\right\}$ are exactly the trajectories of solutions to (12) or (15). Now, we define

$$
L_{k}^{+}=L_{k} \cap\{\psi>0\} .
$$

Notice that if $(\varphi, \psi) \in L_{k}^{+}$is a solution of the system (12), we have

$$
\begin{aligned}
\frac{d k(\xi)}{d \xi} & =\psi \psi^{\prime}+m \varphi^{m+p-1}(1-\varphi)^{q}(\varphi-a) \varphi^{\prime} \\
& =\frac{c}{m} \varphi^{1-m} \psi^{2}+\psi \varphi^{p}(1-\varphi)^{q}\left(\varphi-\varphi_{c \tau}\right) \\
& \geq \frac{c}{m} \varphi^{1-m} \psi^{2} \\
& >0,
\end{aligned}
$$

since $\varphi(\xi)$ is increasing in $\xi$. This implies that $(\varphi, \psi)$ wanders through increasing level sets with increasing $\xi$. See Figure 3(b). Let

$$
k_{1}=m \int_{0}^{1} s^{m+p-1}(1-s)^{q}(s-a) d s
$$

we know that $L_{k_{1}}$ passes through the critical point $(1,0)$. Denote that

$$
\psi^{*}(\varphi)=\sqrt{2 m \int_{\varphi}^{1} s^{m+p-1}(1-s)^{q}(s-a) d s} .
$$

In what follows, we will see that $\psi^{*}(\varphi)$ plays a special role for the proof of the main result. We first need a lemma as follows.

Lemma 4. The trajectory $\psi_{c}(\varphi)$ of the problem (24) must intersect with $\psi^{*}(\varphi)$ for sufficiently large $c>0$. (See Figure 3(c).)

Proof. For any $\varphi \in(0, a]$, we have $\varphi_{c \tau} \in(0, a]$, and

$$
\psi_{c}^{\prime}(\varphi) \geq c>0
$$

Let $\varphi_{0} \in(a, 1)$ be the first point such that $\psi_{c}^{\prime}\left(\varphi_{0}\right)=0$. Then, we have

$$
\psi_{c}\left(\varphi_{0}\right)=\frac{m \varphi_{0}^{m+p-1}\left(1-\varphi_{0}\right)^{q}\left(\varphi_{0 c \tau}-a\right)}{c} .
$$

Since

$$
\psi_{c}\left(\varphi_{0}\right)>\psi_{c}(a) \geq a c
$$

we have

$$
m \varphi_{0}^{m+p-1}\left(1-\varphi_{0}\right)^{q}\left(\varphi_{0 c \tau}-a\right)>a c^{2}
$$

Thus,

$$
m \varphi_{0}^{m+p-1}\left(1-\varphi_{0}\right)^{q}\left(\varphi_{0}-a\right)>a c^{2} .
$$

Denote that

$$
M=\max _{\varphi \in(a, 1)}\left\{\frac{m \varphi^{m+p-1}(1-\varphi)^{q}(\varphi-a)}{a}\right\} .
$$

Then, for any $c \geq \sqrt{M},(74)$ does not hold and $\psi_{c}(\varphi)$ is increasing on $(a, 1)$. Therefore, $\psi_{c}(\varphi)$ must intersect with $\psi^{*}(\varphi)$ for any $c \geq \sqrt{M}$. The proof is completed.

Theorem 5. (i) If $\int_{0}^{1} s^{m+p-1}(1-s)^{q}(s-a) d s \leq 0$, then there is no nontrivial nonnegative solution for problem (23).

(ii) If $\int_{0}^{1} s^{m+p-1}(1-s)^{q}(s-a) d s>0$, then there exists a unique $c_{1}^{*}>0$, such that the problem (23) admits a nonnegative solution $\psi(\varphi)$, and $\psi(\varphi)>0$ for any $\varphi \in(0,1)$.

Proof. (i) The case $\int_{0}^{1} s^{m+p-1}(1-s)^{q}(s-a) d s \leq 0$ has been discussed.

(ii) We know that for any fixed $c>0,\left(\varphi, \psi_{c}\right)$ wanders through increasing level sets $\left\{L_{k}\right\}$ strictly. Thus, $\left(\varphi, \psi_{c}\right)$ 
intersects with a level set $L_{k}$ at most once. Let $\left(\sigma_{c}, \phi_{c}\right)$ be the intersection point of $\left(\varphi, \psi_{c}\right)$ with $L_{k_{1}}$. Then, if $\phi_{c}>0$, we have

$$
\begin{aligned}
\left.\frac{d \psi^{*}}{d \varphi}\right|_{\varphi=\sigma_{c}} & =-\left.\frac{m \varphi^{m+p-1}(1-\varphi)^{q}(\varphi-a)}{\psi^{*}}\right|_{\varphi=\sigma_{c}} \\
& \leq-\left.\frac{m \varphi^{m+p-1}(1-\varphi)^{q}\left(\varphi_{c \tau}-a\right)}{\phi_{c}}\right|_{\varphi=\sigma_{c}} \\
& =\left.\frac{d \psi_{c}}{d \varphi}\right|_{\varphi=\sigma_{c}}-c .
\end{aligned}
$$

Define

$$
c_{1}^{*}
$$

$=\inf \left\{c>0 ; \psi_{c}\right.$ intersects $\psi^{*}$ at $\left(\sigma_{c}, \phi_{c}\right)$ with $\left.\sigma_{c} \in(0,1]\right\}$.

By Lemma $4, c_{1}^{*}$ is well defined. In what follows, we will show that $c_{1}^{*}$ is just the desired wave speed.

We first have $c_{1}^{*}>0$. Indeed, when $c=0$, the first equation of (24) becomes

$$
\frac{d \psi_{0}}{d \varphi}=-\frac{m \varphi^{m+p-1}(1-\varphi)^{q}(\varphi-a)}{\psi_{0}}
$$

Noticing that $\psi_{0}\left(0^{+}\right)=0$, we have

$$
\psi_{0}^{2}(\varphi)=-2 m \int_{0}^{\varphi} s^{m+p-1}(1-s)^{q}(s-a) d s .
$$

Then, there exists $\varphi_{0} \in(a, 1)$ such that

$$
\psi_{0}\left(\varphi_{0}\right)=0
$$

since $\int_{0}^{1} s^{m+p-1}(1-s)^{q}(s-a) d s>0$. The continuous dependence of $\psi_{c}$ on $c$ ensures that $\psi_{c}$ goes to zero before reaching $\psi^{*}$ for sufficiently small $c>0$. Thus, $c_{1}^{*}>0$.

In addition, by Lemma 3, we know that $\sigma_{c}$ is decreasing on $c$. Assume that

$$
\lim _{c \searrow c_{1}^{*}} \sigma_{c}=\sigma_{c_{1}^{*}}
$$

If $\sigma_{c_{1}^{*}}<1$; namely, $\phi_{c_{1}^{*}}>0$, then by (76), we arrive at

$$
\psi_{c_{1}^{*}}^{\prime}\left(\sigma_{c_{1}^{*}}\right) \geq \psi^{*^{\prime}}\left(\sigma_{c_{1}^{*}}\right)+c_{1}^{*}>\psi^{*^{\prime}}\left(\sigma_{c_{1}^{*}}\right) .
$$

So, there exists $\sigma_{c_{1}^{*}}<\varphi_{1}<1$, such that $\psi_{c_{1}^{*}}\left(\varphi_{1}\right)>\psi^{*}\left(\varphi_{1}\right)$. By the continuous dependence of $\psi_{c}$ on $c$, there is $c>0$ with $c<c_{1}^{*}$ and close to $c_{1}^{*}$ sufficiently such that $\psi_{c}\left(\varphi_{1}\right) \geq \psi^{*}\left(\varphi_{1}\right)$, which implies that $\psi_{c}$ intersects with $\psi^{*}$. This contradicts the definition of $c_{1}^{*}$. Thus, $\sigma_{c_{1}^{*}}=1$, which implies $\phi_{c_{1}^{*}}=0$.

Moreover, by Lemma 3, for $c>c_{1}^{*}$, we have $\psi_{c}(1)>0$. However, if $0<c<c_{1}^{*}$, there exists $\varphi_{c}$ with $a<\varphi_{c}<1$ such that $\psi_{c}(\varphi) \rightarrow 0$ as $\varphi \nearrow \varphi_{c}$. The proof is completed.

Proposition 6. $\varphi(\xi)$ is a monotone increasing sharp- or smooth-type traveling wave front of the problem (8)-(9) for some fixed $c>0$, if and only if $\psi(\varphi)$ with $\psi(\varphi)>0$ for any $\varphi \in(0,1)$ is a solution of the problem (23).
Proof. The necessity is clear. Consider the sufficient one. Let $\psi(\varphi)>0$ for any $\varphi \in(0,1)$ be a solution of the problem (23), and $\varphi(\xi)$ solves

$$
\varphi^{\prime}(\xi)=\frac{1}{m} \varphi^{1-m}(\xi) \psi(\varphi(\xi)) .
$$

Without loss of generality, let $\varphi(0)=1 / 2$, and let $(\alpha, \beta)$ be the maximal existence interval of $\varphi$ such that $0<\varphi<1$. Firstly, we have

$$
\begin{aligned}
\left(\varphi^{m}\right)^{\prime \prime}(\xi) & =\psi^{\prime}(\varphi) \varphi^{\prime}(\xi) \\
& =\left(c-\frac{m \varphi^{m+p-1}(1-\varphi)^{q}\left(\varphi_{c \tau}-a\right)}{\psi}\right) \varphi^{\prime} \\
& =c \varphi^{\prime}-\varphi^{p}(1-\varphi)^{q}\left(\varphi_{c \tau}-a\right) .
\end{aligned}
$$

Moreover,

$$
\begin{gathered}
\varphi(\xi)=0 \quad \text { for } \alpha-c \tau<\xi \leq \alpha, \\
\varphi(\beta)=1, \quad\left(\varphi^{m}\right)^{\prime}(\alpha)=\left(\varphi^{m}\right)^{\prime}(\beta)=0 .
\end{gathered}
$$

Therefore, if $\alpha>-\infty$ and $\varphi^{\prime}\left(\alpha^{+}\right) \neq 0, \varphi(\xi)$ is a sharp-type traveling wave front; if $\alpha=-\infty$ or $\varphi^{\prime}\left(\alpha^{+}\right)=0, \varphi(\xi)$ is a smooth-type traveling wave front.

Theorem 5 and Proposition 6 imply the following result.

Theorem 7. (i) If $\int_{0}^{1} s^{m+p-1}(1-s)^{q}(s-a) d s \leq 0$, then there is no increasing traveling wave front for the problem (8)-(9).

(ii) If $\int_{0}^{1} s^{m+p-1}(1-s)^{q}(s-a) d s>0$, then there is a unique wave speed $c_{1}^{*}>0$, such that the problem (8)-(9) admits an increasing traveling wave front.

Furthermore, we have the following results.

Theorem 8. Let $\varphi(\xi)$ be the traveling wave front of the problem (8)-(9) corresponding to the wave speed $c_{1}^{*}$.

(i) If $m<2$, then $\varphi(\xi)$ is a smooth-type traveling wave front.

(ii) If $m=2$, then $\varphi^{\prime}\left(\xi_{a}^{+}\right)=c_{1}^{*} / 2$ and $\varphi(\xi)$ is a sharp-type traveling wave front.

(iii) If $m>2$, then $\varphi^{\prime}\left(\xi_{a}^{+}\right)=+\infty$ and $\varphi(\xi)$ is a sharp-type traveling wave front.

Proof. (i) If $0<m \leq 1$, it is easy to see that

$$
\varphi^{\prime}\left(\xi_{a}^{+}\right)=\frac{1}{m} \varphi^{1-m}\left(\xi_{a}^{+}\right) \psi\left(\xi_{a}^{+}\right)=0 .
$$

If $1<m<2$, from the proof of Lemma 3, we see that when $\varphi>0$ is sufficiently small,

$$
\psi(\varphi)=c_{1}^{*} \varphi+o(\varphi), \quad m+p>2 ;
$$

$\psi(\varphi)=\frac{\sqrt{c_{1}^{* 2}+4 m a}+c_{1}^{*}}{2} \varphi+o(\varphi), \quad m+p=2 ;$

$\psi(\varphi)=\sqrt{\frac{2 m a}{m+p}} \varphi^{(m+p) / 2}+o\left(\varphi^{(m+p) / 2}\right), \quad 1<m+p<2$. 
Since

$$
\varphi^{\prime}(\xi)=\frac{1}{m} \varphi^{1-m}(\xi) \psi(\xi)
$$

we have

$$
\begin{gathered}
\varphi^{\prime}(\xi)=\frac{c_{1}^{*}}{m} \varphi^{2-m}(\xi)+o\left(\varphi^{2-m}(\xi)\right), \quad m+p>2 ; \\
\varphi^{\prime}(\xi)=\frac{\sqrt{c_{1}^{* 2}+4 m a}+c_{1}^{*} \varphi^{2-m}(\xi)+o\left(\varphi^{2-m}(\xi)\right),}{m+p=2 ;} \\
\varphi^{\prime}(\xi)=\sqrt{\frac{2 a}{m(m+p)} \varphi^{(2-m+p) / 2}(\xi)+o\left(\varphi^{(2-m+p) / 2}(\xi)\right),} \\
1<m+p<2 .
\end{gathered}
$$

Thus,

$$
\varphi^{\prime}\left(\xi_{a}^{+}\right)=0
$$

(ii) If $m=2$, we have

$$
\varphi^{\prime}\left(\xi_{a}^{+}\right)=\frac{c_{1}^{*}}{2} .
$$

(iii) If $m>2$, we have

$$
\varphi^{\prime}\left(\xi_{a}^{+}\right)=+\infty .
$$

The proof is completed.

Theorem 9. $c_{1}^{*}(\tau)$ is nonincreasing in delay $\tau$; namely, if $\tau_{1}>$ $\tau_{2}$, then $c_{1}^{*}\left(\tau_{1}\right) \leq c_{1}^{*}\left(\tau_{2}\right)$.

Proof. Suppose for contradiction that there exist time delays $\tau_{1}$ and $\tau_{2}$, with $\tau_{1}>\tau_{2}$ and $c_{1}^{*}\left(\tau_{1}\right)>c_{1}^{*}\left(\tau_{2}\right)$. Denote that $\widehat{c}_{1}=$ $c_{1}^{*}\left(\tau_{1}\right)$ and $\widehat{c}_{2}=c_{1}^{*}\left(\tau_{2}\right)$ for simplicity. Take $c$ such that

$$
\widehat{c}_{2}<c<\widehat{c}_{1} \text {. }
$$

In what follows, denote the solutions of (24) corresponding to wave speed and time delay $\left(\widehat{c}_{1}, \tau_{1}\right),\left(c, \tau_{1}\right),\left(\widehat{c}_{2}, \tau_{2}\right)$ by $\psi_{\widehat{c}_{1}}, \psi_{c}$, $\psi_{\widehat{c}_{2}}$, respectively.

Similar to the proof of Lemma 3, we know that when $\varphi>$ 0 is sufficiently small, for $m+p>2$,

$$
\begin{aligned}
\psi_{c}(\varphi) & =c \varphi+o(\varphi), \\
\psi_{\widehat{c}_{2}}(\varphi) & =\widehat{c}_{2} \varphi+o(\varphi) ;
\end{aligned}
$$

for $m+p=2$,

$$
\begin{gathered}
\psi_{c}(\varphi)=\frac{\sqrt{c^{2}+4 m a}+c}{2} \varphi+o(\varphi), \\
\psi_{\widehat{c}_{2}}(\varphi)=\frac{\sqrt{\widehat{c}_{2}^{2}+4 m a}+\widehat{c}_{2}}{2} \varphi+o(\varphi) ;
\end{gathered}
$$

for $1<m+p<2$,

$$
\begin{aligned}
\psi_{c}^{2}(\varphi)= & \frac{4 c}{m+p+2} \sqrt{\frac{2 m a}{m+p}} \varphi^{(m+p+2) / 2} \\
& +\frac{2 m a}{m+p} \varphi^{m+p}+o\left(\varphi^{(m+p+2) / 2}\right), \\
\psi_{\widehat{c}_{2}}^{2}(\varphi)= & \frac{4 \widehat{c_{2}}}{m+p+2} \sqrt{\frac{2 m a}{m+p}} \varphi^{(m+p+2) / 2} \\
& +\frac{2 m a}{m+p} \varphi^{m+p}+o\left(\varphi^{(m+p+2) / 2}\right) .
\end{aligned}
$$

Since $c>\widehat{c}_{2}$, we have

$$
\psi_{c}(\varphi)>\psi_{\widehat{c_{2}}}(\varphi)
$$

for sufficiently small $\varphi>0$. Furthermore, we claim that $\psi_{c}(\varphi)>\psi_{\widehat{c}_{2}}(\varphi)$ for $\varphi \in(0,1)$. Otherwise, there exists $\varphi_{0} \in$ $(0,1)$ such that $\psi_{c}(\varphi)>\psi_{\widehat{c}_{2}}(\varphi)$ for $\varphi \in\left(0, \varphi_{0}\right)$, and $\psi_{c}\left(\varphi_{0}\right)=$ $\psi_{\widehat{c}_{2}}\left(\varphi_{0}\right)=\psi_{0}$. Then, we have

$$
\psi_{c}^{\prime}\left(\varphi_{0}\right) \leq \psi_{\widehat{c}_{2}}^{\prime}\left(\varphi_{0}\right)
$$

that is,

$$
0<c-\widehat{c}_{2} \leq \frac{m \varphi_{0}^{m+p-1}\left(1-\varphi_{0}\right)^{q}\left(\varphi_{0 c \tau_{1}}-\varphi_{0 \widehat{c}_{2} \tau_{2}}\right)}{\psi_{0}}
$$

which implies $\varphi_{0 c \tau_{1}}>\varphi_{0 \widehat{c}_{2} \tau_{2}}$. On the other hand, by Lemma 2, we have $\varphi_{0 c \tau_{1}} \leq \varphi_{0 \widehat{c}_{2} \tau_{2}}$, a contradiction. Similarly, we can get $\psi_{\widehat{c}_{1}}(\varphi)>\psi_{c}(\varphi)$. From the uniqueness of wave speed on any fixed $\tau>0$, this contradicts the definition of $\widehat{c}_{1}$.

\section{Existence of Decreasing Traveling Waves}

In this section, we aim to find a solution $\psi(\varphi)$ of the problem (23) with $\psi(\varphi)<0$ for $\varphi \in(0,1)$. We first introduce a comparison lemma.

Lemma 10. Let $u, v$ be the solutions of the following problems, respectively:

$$
\begin{array}{ll}
\frac{d u}{d \varphi}=c-\frac{m \varphi^{m+p^{-1}}(1-\varphi)^{q}(\varphi-a)}{u} & \text { for } \varphi<1, u\left(1^{-}\right)=0, \\
\frac{d v}{d \varphi}=c-\frac{m \varphi^{m+p^{-1}}(1-\varphi)^{q}(1-a)}{v} & \text { for } \varphi<1, v\left(1^{-}\right)=0 .
\end{array}
$$

And let $\psi$ solve the problem (23). Then, $\psi(\varphi) \leq u(\varphi) \leq v(\varphi)$ for 
$\varphi<1$ if $\psi, u, v$ are positive, while $v(\varphi) \leq \psi(\varphi) \leq u(\varphi)$ for $\varphi<1$ if $\psi, u, v$ are negative.

Proof. Notice that $\varphi_{c \tau} \leq \varphi$ when $\psi$ is positive, and thus

$$
\begin{aligned}
\frac{1}{2} \frac{d \psi^{2}}{d \varphi}-c \psi & =-m \varphi^{m+p-1}(1-\varphi)^{q}\left(\varphi_{c \tau}-a\right) \\
& \geq-m \varphi^{m+p-1}(1-\varphi)^{q}(\varphi-a) \\
& =\frac{1}{2} \frac{d u^{2}}{d \varphi}-c u ;
\end{aligned}
$$

that is,

$$
\frac{d\left(\psi^{2}-u^{2}\right)}{d \varphi}-2 c\left(\psi^{2}-u^{2}\right) g(\varphi) \geq 0
$$

where

$$
g(\varphi)=\frac{1}{\psi+u}
$$

Consequently,

$$
\frac{d\left(\left(\psi^{2}-u^{2}\right) e^{2 c \int_{\varphi}^{1} g(s) d s}\right)}{d \varphi} \geq 0 .
$$

Integrating from $\varphi$ to 1 yields

$$
\psi^{2}(\varphi)-u^{2}(\varphi) \leq 0
$$

and so $\psi(\varphi) \leq u(\varphi)$ if $\psi, u>0$. Similarly, $u(\varphi) \leq v(\varphi)$ if $u, v>0$.

The proof for $\psi, u, v<0$ is similar and omitted here.

Since $\psi<0$, we see that $\varphi(\xi)$ is decreasing in $\xi$, and so $\varphi_{c \tau}(\xi) \geq \varphi(\xi)$. To get the behavior of the trajectories $\psi_{c}(\varphi)$ of (23), we have to study the trajectories starting from $(1,0)$, since the property of $\psi_{c}$ at $\varphi$ depends on the behavior of $\psi_{c}$ at $\varphi_{c \tau}$ closely. Consider the following problem:

$$
\begin{gathered}
\frac{d \psi}{d \varphi}=c-\frac{m \varphi^{m+p-1}(1-\varphi)^{q}\left(\varphi_{c \tau}-a\right)}{\psi} \text { for } \varphi \in(\alpha, 1), \\
\psi\left(1^{-}\right)=0 \\
\psi(\varphi)<0 \quad \text { for } \varphi \in(\alpha, 1)
\end{gathered}
$$

where $(\alpha, 1)$ with $\alpha \geq 0$ is the maximal existence interval of the solution $\psi(\varphi)<0$. By $(106)$,

$$
\begin{aligned}
\frac{1}{2} \psi^{2}(\varphi) & =-\int_{\varphi}^{1} c \psi(s) d s+m \int_{\varphi}^{1} s^{m+p-1}(1-s)^{q}\left(s_{c \tau}-a\right) d s \\
& \geq-\int_{\varphi}^{1} c \psi(s) d s+m \int_{\varphi}^{1} s^{m+p-1}(1-s)^{q}(s-a) d s
\end{aligned}
$$

Hence, $\psi\left(0^{+}\right)=0$ is impossible if $\int_{0}^{1} s^{m+p-1}(1-s)^{q}(s-a) d s \geq$ 0 . So, we only need to find the decreasing traveling wave fronts for the case $\int_{0}^{1} s^{m+p-1}(1-s)^{q}(s-a) d s<0$. We first give the following three lemmas.

Lemma 11. Assume that $0<\varphi_{0}<1$ and $\psi_{1}(\varphi), \psi_{2}(\varphi)$ are solutions of (106) corresponding to different wave speeds $c_{1}, c_{2}$, respectively, where $\psi_{1}\left(\varphi_{0}\right) \leq \psi_{2}\left(\varphi_{0}\right)<0, \psi_{1}(\varphi)<\psi_{2}(\varphi)<0$ for $\varphi_{0}<\varphi<1$, and $\varphi_{1}\left(\xi_{1}\right)=\varphi_{2}\left(\xi_{2}\right)=\varphi_{0}$. Then, $\varphi_{1}\left(\xi_{1}-s\right)>$ $\varphi_{2}\left(\xi_{2}-s\right)$ if $0<s<\int_{\varphi_{0}}^{1}\left(m \sigma^{m-1} /-\psi_{2}(\sigma)\right) d \sigma$, and $\varphi_{1}\left(\xi_{1}-s\right)=$ $\varphi_{2}\left(\xi_{2}-s\right)=1$ if $s \geq \int_{\varphi_{0}}^{1}\left(m \sigma^{m-1} /-\psi_{2}(\sigma)\right) d \sigma$.

Proof. The proof is similar to that of Lemma 2.

Lemma 12. For any given $\tau>0$, and $c_{1}>c_{2} \geq 0$, let $\psi_{1}(\varphi)$, $\psi_{2}(\varphi)$ be solutions of (106) corresponding to $c_{1}, c_{2}$, respectively. Then, $\psi_{1}(\varphi)<\psi_{2}(\varphi)$ for any $\varphi \in\left(\alpha_{2}, 1\right)$, where $\left(\alpha_{2}, 1\right)$ is the maximal existence interval of the solution $\psi_{2}(\varphi)<0$. In addition, $\psi_{1}\left(\alpha_{2}\right)<0$. (See Figure $4(a)$.)

Proof. We first show that $\psi_{1}(\varphi)<\psi_{2}(\varphi)$ if $\varphi$ is in a sufficiently small left neighborhood of 1 . The argument consists of three cases, $q>1, q=1$, and $q<1$.

(i) Consider the case $q>1$. According to (106), we have

$$
\frac{d \psi}{d \varphi}=c-\frac{m \varphi^{m+p-1}(1-\varphi)^{q}\left(\varphi_{c \tau}-a\right)}{\psi} .
$$

Noticing that $\varphi_{c \tau} \geq \varphi$, we have for $\varphi \geq a$ that

$$
\frac{d \psi}{d \varphi} \geq c
$$

Integrating from $\varphi$ to 1 yields

$$
-\psi(\varphi) \geq c(1-\varphi) .
$$

We further have

$$
\begin{aligned}
\frac{d \psi}{d \varphi} & =c-\frac{m \varphi^{m+p-1}(1-\varphi)^{q}\left(\varphi_{c \tau}-a\right)}{\psi} \\
& \leq c+\frac{m \varphi^{m+p-1}(1-\varphi)^{q}\left(\varphi_{c \tau}-a\right)}{c(1-\varphi)} \\
& \leq c+\frac{m(1-a)}{c}(1-\varphi)^{q-1} .
\end{aligned}
$$

Integrating from $\varphi$ to 1 gives

$$
-\psi(\varphi) \leq c(1-\varphi)+\frac{m(1-a)}{c q}(1-\varphi)^{q} .
$$

Thus,

$$
\psi(\varphi) \sim c(\varphi-1), \quad \text { as } \varphi \longrightarrow 1^{-} .
$$

Therefore, $\psi_{1}(\varphi)<\psi_{2}(\varphi)$ in a left neighborhood of $\varphi=1$. 


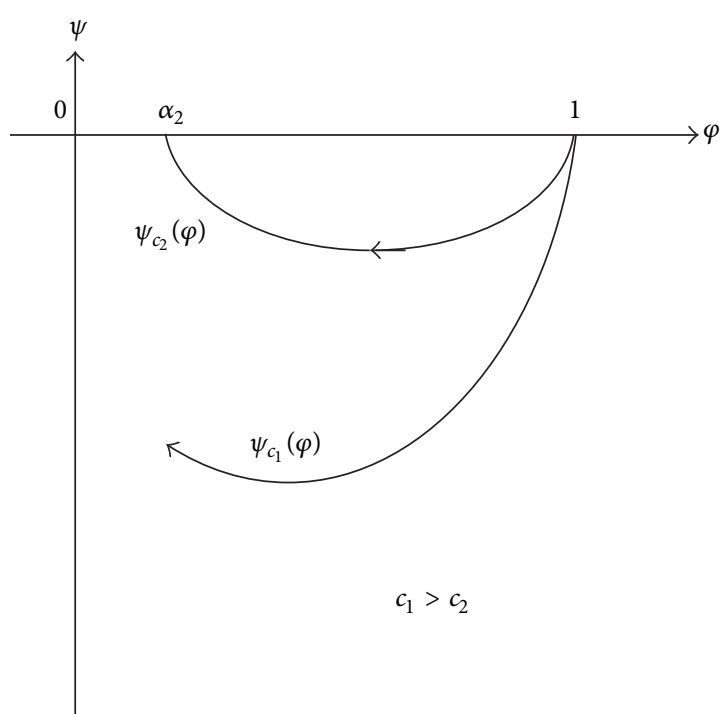

(a)

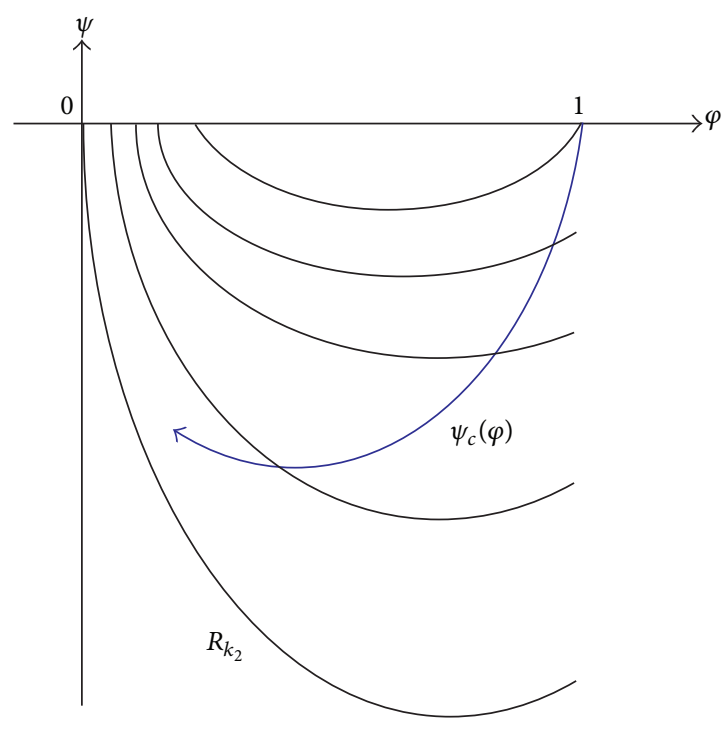

(b)

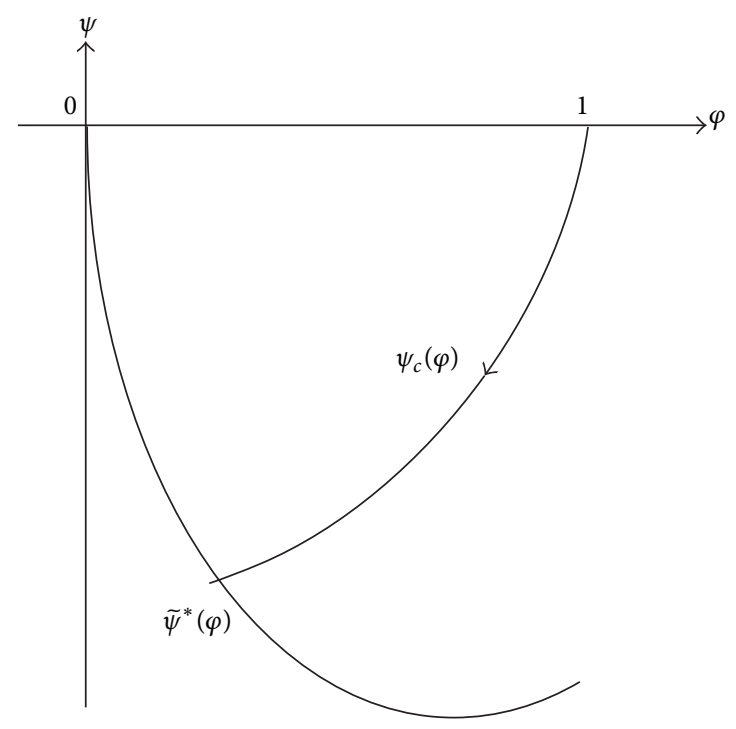

(c)

Figure 4: The properties of the trajectory $\psi_{c}(\varphi)$. (a) The monotonicity of $\psi_{c}(\varphi)$ on $c$. (b) The trajectory $\psi_{c}(\varphi)$ wanders through $R_{k}$. (c) The trajectory $\psi_{c}(\varphi)$ intersects with $\widetilde{\psi}^{*}(\varphi)$ for large $c$.

(ii) When $q=1$, consider the following two systems:

$$
\begin{gathered}
\varphi^{\prime}(\xi)=\frac{1}{m} \varphi^{1-m}(\xi) u_{1}(\xi), \\
u_{1}^{\prime}(\xi)=\frac{c}{m} \varphi^{1-m}(\xi) u_{1}(\xi)-\varphi(\xi)^{p}(1-\varphi(\xi))(\varphi(\xi)-a), \\
\varphi^{\prime}(\xi)=\frac{1}{m} \varphi^{1-m}(\xi) u_{2}(\xi), \\
u_{2}^{\prime}(\xi)=\frac{c}{m} \varphi^{1-m}(\xi) u_{2}(\xi)-\varphi(\xi)^{p}(1-\varphi(\xi))(1-a) .
\end{gathered}
$$

Notice that the right hand side of the previous two systems shares the same Jacobian matrix

$$
P=\left(\begin{array}{cc}
0 & \frac{1}{m} \\
1-a & \frac{c}{m}
\end{array}\right)
$$

at $(1,0)$. By a simple calculation, we get the eigenvalues of the matrix $P$ as

$\lambda_{+}=\frac{c+\sqrt{c^{2}+4 m(1-a)}}{2 m}, \quad \lambda_{-}=\frac{c-\sqrt{c^{2}+4 m(1-a)}}{2 m}$. 
It is easy to see that $(1,0)$ is a saddle point. And the eigenvector associated with the eigenvalue $\lambda_{+}$can be

$$
Q=\left(\begin{array}{c}
1 \\
m \lambda_{+}
\end{array}\right) \text {. }
$$

We express the local explicit solutions of the problem (114) in the left neighborhood of $(1,0)$ to reach

$$
\begin{gathered}
\varphi(\xi)=1-l e^{\lambda_{+} \xi}+\mathcal{O}\left((1-\varphi)^{2}\right), \\
u_{i}(\xi)=-\operatorname{lm} \lambda_{+} e^{\lambda_{+} \xi}+\mathcal{O}\left(u_{i}^{2}\right), \quad i=1,2 .
\end{gathered}
$$

Therefore, near $(1,0)$, we have

$$
u_{i} \sim m \lambda_{+}(\varphi-1)=\frac{c+\sqrt{c^{2}+4 m(1-a)}}{2}(\varphi-1), \quad i=1,2 .
$$

By the comparison lemma, $u_{2} \leq \psi \leq u_{1}$, which implies

$$
\psi(\varphi) \sim \frac{c+\sqrt{c^{2}+4 m(1-a)}}{2}(\varphi-1), \quad \text { as } \varphi \longrightarrow 1^{-} .
$$

Thus, $\psi_{1}(\varphi)<\psi_{2}(\varphi)$ in a left neighborhood of $\varphi=1$.

(iii) Consider the case $0<q<1$. Notice that

$$
\frac{d \psi}{d \varphi} \geq \frac{m \varphi^{m+p-1}(1-\varphi)^{q}(a-\varphi)}{\psi},
$$

which means that

$$
\begin{aligned}
-\psi^{2}(\varphi) & \leq \int_{\varphi}^{1} 2 m s^{m+p-1}(1-s)^{q}(a-s) d s \\
& =\frac{2 m(a-1)}{q+1}(1-\varphi)^{q+1}+o\left((1-\varphi)^{q+1}\right) ;
\end{aligned}
$$

that is,

$$
-\psi(\varphi) \geq \sqrt{\frac{2 m(1-a)}{q+1}}(1-\varphi)^{(q+1) / 2}+o\left((1-\varphi)^{(q+1) / 2}\right) .
$$

Consequently,

$$
\begin{aligned}
\frac{d \psi}{d \varphi} & \leq c-\frac{m(1-a)(1-\varphi)^{q}}{\psi} \\
& \leq c+\frac{m(1-a)(1-\varphi)^{q}}{\sqrt{2 m(1-a) /(q+1)}(1-\varphi)^{(q+1) / 2}+o\left((1-\varphi)^{(q+1) / 2}\right)} \\
& =c+\sqrt{\frac{m(1-a)(q+1)}{2}}(1-\varphi)^{(q-1) / 2}+o\left((1-\varphi)^{(q-1) / 2}\right) .
\end{aligned}
$$

That is,

$$
\begin{aligned}
-\psi(\varphi) \leq & c(1-\varphi)+\sqrt{\frac{2 m(1-a)}{q+1}}(1-\varphi)^{(q+1) / 2} \\
& +o\left((1-\varphi)^{(q+1) / 2}\right) .
\end{aligned}
$$

Thus, we have

$$
-\psi(\varphi)=\sqrt{\frac{2 m(1-a)}{q+1}}(1-\varphi)^{(q+1) / 2}+o\left((1-\varphi)^{(q+1) / 2}\right) .
$$

Recalling (123), we see that

$$
\begin{aligned}
&-\frac{d \psi^{2}}{d \varphi}=-2 c \psi+2 m \varphi^{m+p-1}(1-\varphi)^{q}\left(\varphi_{c \tau}-a\right) \\
& \geq 2 c \sqrt{\frac{2 m(1-a)}{q+1}}(1-\varphi)^{(q+1) / 2} \\
& \quad+2 m \varphi^{m+p-1}(1-\varphi)^{q}(\varphi-a)+o\left((1-\varphi)^{(q+1) / 2}\right),
\end{aligned}
$$

which implies that

$$
\begin{aligned}
\psi^{2}(\varphi) \geq & \frac{4 c}{q+3} \sqrt{\frac{2 m(1-a)}{q+1}}(1-\varphi)^{(q+3) / 2} \\
& +\frac{2 m(1-a)}{q+1}(1-\varphi)^{q+1}+o\left((1-\varphi)^{q+1}\right) .
\end{aligned}
$$

On the other hand, by (126), we have

$$
\begin{aligned}
-\frac{d \psi^{2}}{d \varphi} \leq & -2 c \psi+2 m(1-a)(1-\varphi)^{q} \\
= & 2 c \sqrt{\frac{2 m(1-a)}{q+1}}(1-\varphi)^{(q+1) / 2}+2 m(1-a)(1-\varphi)^{q} \\
& +o\left((1-\varphi)^{(q+1) / 2}\right),
\end{aligned}
$$

and, hence,

$$
\begin{aligned}
\psi^{2}(\varphi) \leq & \frac{4 c}{q+3} \sqrt{\frac{2 m(1-a)}{q+1}}(1-\varphi)^{(q+3) / 2} \\
& +\frac{2 m(1-a)}{q+1}(1-\varphi)^{q+1}+o\left((1-\varphi)^{q+1}\right) .
\end{aligned}
$$

Summing up, we arrive at

$$
\begin{aligned}
& \psi^{2}(\varphi)= \frac{4 c}{q+3} \sqrt{\frac{2 m(1-a)}{q+1}}(1-\varphi)^{(q+3) / 2} \\
&+\frac{2 m(1-a)}{q+1}(1-\varphi)^{q+1}+o\left((1-\varphi)^{q+1}\right), \\
& \text { as } \varphi \longrightarrow 1^{-},
\end{aligned}
$$

which implies that $\psi_{1}(\varphi)<\psi_{2}(\varphi)$ in a left neighborhood of $\varphi=1$.

The proof for the claims that $\psi_{1}(\varphi)<\psi_{2}(\varphi)$ for any $\varphi \in$ $\left(\alpha_{2}, 1\right)$ and $\psi_{1}\left(\alpha_{2}\right)<0$ is similar to the proof of Lemma 3 and omitted here. 
Similar to Section 2, we denote level set $R_{k}$ by

$$
\begin{aligned}
& R_{k} \\
& =\left\{(\varphi, \psi) \in \mathbb{R}^{2} ; \frac{1}{2} \psi^{2}-m \int_{\varphi}^{1} s^{m+p-1}(1-s)^{q}(s-a) d s=k\right\}
\end{aligned}
$$

for any $k \geq 0$, and correspondingly, define

$$
R_{k}^{-}=R_{k} \cap\{\psi<0\} \text {. }
$$

Notice that if $(\varphi, \psi) \in R_{k}^{-}$solves system (12), then

$$
\begin{aligned}
\frac{d k(\xi)}{d \xi} & =\psi \psi^{\prime}+m \varphi^{m+p-1}(1-\varphi)^{q}(\varphi-a) \varphi^{\prime} \\
& =\frac{c}{m} \varphi^{1-m} \psi^{2}+\psi \varphi^{p}(1-\varphi)^{q}\left(\varphi-\varphi_{c \tau}\right) \\
& \geq \frac{c}{m} \varphi^{1-m} \psi^{2} \\
& >0
\end{aligned}
$$

since $\varphi(\xi)$ is decreasing in $\xi$. This implies that the trajectory $(\varphi, \psi)$ of (106) wanders through increasing level sets with increasing $\xi$. See Figure 4(b). Letting

$$
k_{2}=-m \int_{0}^{1} s^{m+p-1}(1-s)^{q}(s-a) d s,
$$

we know that $R_{k_{2}}$ passes through the critical point $(0,0)$. Denote that

$$
\widetilde{\psi}^{*}(\varphi)=-\sqrt{-2 m \int_{0}^{\varphi} s^{m+p-1}(1-s)^{q}(s-a) d s} .
$$

We introduce the following lemma.

Lemma 13. The trajectory $\psi_{c}(\varphi)$ of the problem (106) must intersect with $\widetilde{\psi}^{*}(\varphi)$ for sufficiently large $c>0$. (See Figure 4(c).)

Proof. For any $\varphi \in[a, 1)$, we have $\varphi_{c \tau} \in[a, 1)$ and

$$
\psi_{c}^{\prime}(\varphi) \geq c>0 .
$$

Let $\varphi_{0} \in(0, a)$ be the first point such that $\psi_{c}^{\prime}\left(\varphi_{0}\right)=0$. Then, we have

$$
\psi_{c}\left(\varphi_{0}\right)=\frac{m \varphi_{0}^{m+p-1}\left(1-\varphi_{0}\right)^{q}\left(\varphi_{0 c \tau}-a\right)}{c} .
$$

Since

$$
\psi_{c}\left(\varphi_{0}\right)<\psi_{c}(a) \leq(a-1) c
$$

we have

$$
m \varphi_{0}^{m+p-1}\left(1-\varphi_{0}\right)^{q}\left(\varphi_{0 c \tau}-a\right)<(a-1) c^{2} .
$$

Thus,

$$
m \varphi_{0}^{m+p-1}\left(1-\varphi_{0}\right)^{q}\left(\varphi_{0}-a\right)<(a-1) c^{2} .
$$

Denote that

$$
\widetilde{M}=\max _{\varphi \in(0, a)}\left\{\frac{m \varphi^{m+p-1}(1-\varphi)^{q}(a-\varphi)}{1-a}\right\} .
$$

Then, for any $c \geq \sqrt{\widetilde{M}}$, (141) does not hold, and $\psi_{c}(\varphi)$ is increasing on $(0, a)$. Therefore, $\psi_{c}(\varphi)$ must intersect with $\widetilde{\psi}^{*}(\varphi)$ for any $c \geq \sqrt{\widetilde{M}}$. The proof is completed.

Theorem 14. (i) If $\int_{0}^{1} s^{m+p-1}(1-s)^{q}(s-a) d s \geq 0$, then there is no nontrivial nonpositive solution for the problem (23).

(ii) If $\int_{0}^{1} s^{m+p-1}(1-s)^{q}(s-a) d s<0$, then there exists a unique $c_{2}^{*}>0$, such that the problem (23) admits a nonpositive solution $\psi(\varphi)$, and $\psi(\varphi)<0$ for any $\varphi \in(0,1)$.

Proof. The proof is similar to that of Theorem 5, and $c_{2}^{*}$

$=\inf \left\{c>0 ; \psi_{c}\right.$ intersects $\widetilde{\psi}^{*}$ at $\left(\eta_{c}, \tilde{\phi}_{c}\right)$ with $\left.\eta_{c} \in[0,1)\right\}$.

Proposition 15. $\varphi(\xi)$ is a monotone decreasing sharp-or smooth-type traveling wave front of the problem (10)-(11) for some fixed $c>0$, if and only if $\psi(\varphi)$ with $\psi(\varphi)<0$ for any $\varphi \in(0,1)$ is a solution of the problem (23).

Proof. The proof is similar to that of Proposition 6.

Theorem 14 and Proposition 15 imply the following result.

Theorem 16. (i) If $\int_{0}^{1} s^{m+p-1}(1-s)^{q}(s-a) d s \geq 0$, then there is no decreasing traveling wave front for the problem (10)-(11).

(ii) If $\int_{0}^{1} s^{m+p-1}(1-s)^{q}(s-a) d s<0$, then there is a unique wave speed $c_{2}^{*}>0$, such that the problem (10)-(11) admits a decreasing traveling wave front.

Furthermore, we have the following results.

Theorem 17. The traveling wave front $\varphi(\xi)$ of the problem (10)(11) corresponding to the wave speed $c_{2}^{*}$ obtained earlier is of smooth type.

Proof. It is easy to see that

$$
\frac{d \psi}{d \varphi} \leq 0, \quad \text { as } \varphi \longrightarrow 0^{+} .
$$

Thus,

$$
\begin{aligned}
\psi & \geq \frac{m \varphi^{m+p-1}(1-\varphi)^{q}\left(\varphi_{c_{2}^{*} \tau}-a\right)}{c_{2}^{*}} \\
& \geq-\frac{m a}{c_{2}^{*}} \varphi^{m+p-1} .
\end{aligned}
$$


Recalling that

$$
\varphi^{\prime}(\xi)=\frac{1}{m} \varphi^{1-m}(\xi) \psi(\xi)
$$

we know that

$$
-\frac{a}{c_{2}^{*}} \varphi^{p}(\xi) \leq \varphi^{\prime}(\xi) \leq 0,
$$

which means that

$$
\varphi^{\prime}\left(\widetilde{\xi}_{b}^{-}\right)=0
$$

The proof is completed.

Theorem 18. $c_{2}^{*}(\tau)$ is nonincreasing in delay $\tau$; namely, if $\tau_{1}>$ $\tau_{2}$, then $c_{2}^{*}\left(\tau_{1}\right) \leq c_{2}^{*}\left(\tau_{2}\right)$.

Proof. The proof is similar to that of Theorem 9.

\section{Asymptotic Behavior of the Traveling Wave Solutions}

In this section, we first pay our attention to the finiteness of $\xi_{a}, \xi_{b}, \widetilde{\xi}_{a}, \widetilde{\xi}_{b}$. On the basis of this, the convergent rates of $\varphi$ going to the steady states at far-field are discussed.

Theorem 19. Let $\varphi(\xi)$ be the increasing traveling wave solution of the problem (8)-(9) corresponding to the unique wave speed $c_{1}^{*}$.

(i) If $0<q<1$, then $\xi_{b}<+\infty$.

(ii) If $q \geq 1$, then $\xi_{b}=+\infty$. More precisely,

(a) when $q>1$, one has

$$
1-\varphi(\xi) \sim\left(\frac{(1-a)(q-1)}{c_{1}^{*}} \xi\right)^{1 /(1-q)}, \quad \text { as } \xi \longrightarrow+\infty
$$

(b) when $q=1$, one has

$$
1-\varphi(\xi) \sim e^{-\left(\left(\sqrt{c_{1}^{* 2}+4 m(1-a)}-c_{1}^{*}\right) / 2 m\right) \xi}, \quad \text { as } \xi \longrightarrow+\infty .
$$

Proof. (i) For $(1+a) / 2 \leq \varphi_{c_{1}^{*} \tau}<1$, we have $(1+a) / 2 \leq \varphi_{c_{1}^{*} \tau} \leq$ $\varphi \leq 1$, and

$$
\begin{aligned}
\frac{1}{2} \frac{d \psi^{2}}{d \varphi}-c_{1}^{*} \psi & =-m \varphi^{m+p-1}(1-\varphi)^{q}\left(\varphi_{c_{1}^{*} \tau}-a\right) \\
& \leq-\frac{m(1-a)}{2}\left(\frac{1+a}{2}\right)^{m+p-1}(1-\varphi)^{q} .
\end{aligned}
$$

Consider the following inequality problem:

$$
\begin{gathered}
\frac{1}{2} \frac{d u^{2}}{d \varphi}-c_{1}^{*} u \geq-\frac{m(1-a)}{2}\left(\frac{1+a}{2}\right)^{m+p-1}(1-\varphi)^{q}, \\
\qquad \text { for } \varphi \leq 1, \\
u\left(1^{-}\right)=0, \\
u(\varphi)>0, \text { for } \varphi<1 .
\end{gathered}
$$

It is easy to prove that $\psi(\varphi) \geq u(\varphi)$ when $\varphi_{c_{1}^{*} \tau} \geq(1+a) / 2$. Now, we construct a function $u$ satisfying (152). Let

$$
u=A(1-\varphi)^{\theta}
$$

Then, $u$ satisfies (152) if and only if

$$
\begin{gathered}
A^{2} \theta(1-\varphi)^{2 \theta-1-q}+c_{1}^{*} A(1-\varphi)^{\theta-q} \\
\leq \frac{m(1-a)}{2}\left(\frac{1+a}{2}\right)^{m+p-1} .
\end{gathered}
$$

Take

$$
\theta=\max \left\{\frac{1+q}{2}, q\right\}
$$

Then, (154) holds when $A$ is appropriately small. Thus,

$$
\psi(\varphi) \geq A(1-\varphi)^{\theta}
$$

with $\theta=\max \{(1+q) / 2, q\}$ and $A$ appropriately small. Since $\varphi(\xi)$ is the solution of the problem (8)-(9), there exists $\varphi_{0}<1$ with $1-\varphi_{0}$ small enough such that when $\varphi_{0}<\varphi<1$,

$$
\begin{aligned}
\xi(\varphi)-\xi\left(\varphi_{0}\right) & =\int_{\varphi_{0}}^{\varphi} \frac{m s^{m-1}}{\psi(s)} d s \\
& \leq \frac{m}{A} \int_{\varphi_{0}}^{\varphi} \frac{s^{m-1}}{(1-s)^{\theta}} d s .
\end{aligned}
$$

When $0<q<1, \xi(\varphi)<+\infty$ as $\varphi \rightarrow 1^{-}$. Thus, $\xi_{b}<+\infty$.

(ii) It is easy to see that

$$
\frac{d \psi}{d \varphi} \leq 0, \quad \text { as } \varphi \longrightarrow 1^{-}
$$

Thus,

$$
\begin{aligned}
\psi & \leq \frac{m \varphi^{m+p-1}(1-\varphi)^{q}\left(\varphi_{c_{1}^{*} \tau}-a\right)}{c_{1}^{*}} \\
& \leq \frac{m \varphi^{m+p-1}(1-\varphi)^{q}(\varphi-a)}{c_{1}^{*}}, \\
\xi(\varphi)-\xi\left(\varphi_{0}\right) & =\int_{\varphi_{0}}^{\varphi} \frac{m s^{m-1}}{\psi(s)} d s \\
& \geq \int_{\varphi_{0}}^{\varphi} \frac{c_{1}^{*}}{s^{p}(1-s)^{q}(s-a)} d s .
\end{aligned}
$$

Letting $\varphi \rightarrow 1$, we obtain

$$
\lim _{\varphi \rightarrow 1^{-}} \xi(\varphi)=+\infty \text {; }
$$

that is,

$$
\xi_{b}=+\infty .
$$


For any $\varepsilon>0$, when $\varphi$ approaches 1 enough, we have $\varphi_{c_{1}^{*} \tau}>1-\varepsilon$. Consider the following two problems:

$$
\begin{gathered}
\frac{1}{2} \frac{d u^{2}}{d \varphi}-c_{1}^{*} u \leq-m \varphi^{m+p-1}(1-\varphi)^{q}(1-a), \\
\text { for } \varphi \leq 1, \\
u\left(1^{-}\right)=0, \\
u(\varphi)>0 \text { for } \varphi<1, \\
\frac{1}{2} \frac{d v^{2}}{d \varphi}-c_{1}^{*} v \geq-m \varphi^{m+p-1}(1-\varphi)^{q}(1-\varepsilon-a), \\
v\left(1^{-}\right)=0, \\
v(\varphi)>0 \text { for } \varphi \leq 1,
\end{gathered}
$$

It is easy to prove that $v(\varphi) \leq \psi(\varphi) \leq u(\varphi)$ when $\varphi<1$ with $\varphi_{c_{1}^{*} \tau}>1-\varepsilon$. In what follows, we construct two functions $u$ and $v$ satisfying (162) and (163), respectively. Let

$$
u=A(1-\varphi)^{\theta}
$$

Then, $u$ satisfies (162) if and only if

$$
A^{2} \theta(1-\varphi)^{2 \theta-1-q}+c_{1}^{*} A(1-\varphi)^{\theta-q} \geq m(1-a) \varphi^{m+p-1} .
$$

When $q>1$, take

$$
\theta=q, \quad A=\frac{m(1-a)}{c_{1}^{*}} .
$$

When $q=1$, take

$$
\begin{gathered}
\theta=1 \\
A=\frac{\sqrt{c_{1}^{* 2}+4 m(1-a)}-c_{1}^{*}}{2} .
\end{gathered}
$$

Then, $u$ is a solution of problem (162).

On the other hand, let

$$
v=\widetilde{A}(1-\varphi)^{\tilde{\theta}} .
$$

Then, $v$ satisfies (163) if and only if

$$
\widetilde{A}^{2} \widetilde{\theta}(1-\varphi)^{2 \widetilde{\theta}-1-q}+c_{1}^{*} \widetilde{A}(1-\varphi)^{\widetilde{\theta}-q} \leq m(1-\varepsilon-a) \varphi^{m+p-1} .
$$

When $q>1$, take $\widetilde{\theta}=q$; then, (169) is ensured by

$$
\begin{aligned}
& \widetilde{A}^{2} q \varepsilon^{q-1}+c_{1}^{*} \widetilde{A} \\
& \quad \leq m(1-\varepsilon-a)(1-\varepsilon)^{m+p-1}\left(1-\varepsilon^{(q-1) / 2}+\varepsilon^{(q-1) / 2}\right)
\end{aligned}
$$

which holds if

$$
\begin{gathered}
\widetilde{A}^{2} q \varepsilon^{(q-1) / 2} \leq m(1-\varepsilon-a)(1-\varepsilon)^{m+p-1}, \\
c_{1}^{*} \widetilde{A} \leq m(1-\varepsilon-a)(1-\varepsilon)^{m+p-1}\left(1-\varepsilon^{(q-1) / 2}\right) .
\end{gathered}
$$

Take

$$
\widetilde{A}=\frac{m(1-\varepsilon-a)(1-\varepsilon)^{m+p-1}\left(1-\varepsilon^{(q-1) / 2}\right)}{c_{1}^{*}} .
$$

Then, when

$$
\varepsilon \leq \min \left\{1-a, \frac{c_{1}^{* 4 /(q-1)}}{(m(1-a))^{2 /(q-1)} q^{2 /(q-1)}}\right\},
$$

(169) holds. When $q=1$, take $\widetilde{\theta}=1$, and

$$
\widetilde{A}=\frac{\sqrt{c_{1}^{* 2}+4 m(1-\varepsilon-a)(1-\varepsilon)^{m+p-1}}-c_{1}^{*}}{2} .
$$

Then, (169) is ensured.

Summing up, for any $\varphi<1$ with $\varphi_{c_{1}^{*} \tau}>1-\varepsilon$, when $q>1$,

$$
\begin{gathered}
\frac{m(1-\varepsilon-a)(1-\varepsilon)^{m+p-1}\left(1-\varepsilon^{(q-1) / 2}\right)}{c_{1}^{*}}(1-\varphi)^{q} \\
\leq \psi(\varphi) \leq \frac{m(1-a)}{c_{1}^{*}}(1-\varphi)^{q} .
\end{gathered}
$$

Noticing that

$$
\xi(\varphi)=\xi\left(\varphi_{0}\right)+\int_{\varphi_{0}}^{\varphi} \frac{m s^{m-1}}{\psi(s)} d s,
$$

we have

$$
\begin{aligned}
& \frac{c_{1}^{*}}{(1-a)(q-1)} \\
& \quad \leq \lim _{\varphi \rightarrow 1^{-}} \xi(\varphi)(1-\varphi)^{q-1} \\
& \quad \leq \frac{c_{1}^{*}}{(1-\varepsilon-a)(1-\varepsilon)^{m+p-1}\left(1-\varepsilon^{(q-1) / 2}\right)(q-1)}
\end{aligned}
$$

that is,

$$
\begin{aligned}
& \left(\frac{(1-a)(q-1)}{c_{1}^{*}}\right)^{1 /(1-q)} \\
& \quad \leq \lim _{\xi \rightarrow+\infty}(1-\varphi(\xi)) \xi^{1 /(q-1)} \\
& \quad \leq\left(\frac{(1-\varepsilon-a)(1-\varepsilon)^{m+p-1}\left(1-\varepsilon^{(q-1) / 2}\right)(q-1)}{c_{1}^{*}}\right)^{1 /(1-q)}
\end{aligned}
$$


When $q=1$,

$$
\begin{aligned}
& \frac{\sqrt{c_{1}^{* 2}+4 m(1-\varepsilon-a)(1-\varepsilon)^{m+p-1}}-c_{1}^{*}}{2}(1-\varphi) \\
& \leq \psi(\varphi) \\
& \leq \frac{\sqrt{c_{1}^{* 2}+4 m(1-a)}-c_{1}^{*}}{2}(1-\varphi) .
\end{aligned}
$$

A direct calculation gives

$$
\begin{aligned}
& -\frac{2 m}{\sqrt{c_{1}^{* 2}+4 m(1-a)}-c_{1}^{*}} \\
& \leq \lim _{\varphi \rightarrow 1^{-}} \frac{\xi(\varphi)}{\ln (1-\varphi)} \\
& \leq-\frac{2 m}{\sqrt{c_{1}^{* 2}+4 m(1-\varepsilon-a)(1-\varepsilon)^{m+p-1}}-c_{1}^{*}} ;
\end{aligned}
$$

that is,

$$
\begin{aligned}
& e^{-\left(\left(\sqrt{c_{1}^{* 2}+4 m(1-a)}-c_{1}^{*}\right) / 2 m\right) \xi} \\
& \quad \leq 1-\varphi(\xi) \\
& \quad \leq e^{-\left(\left(\sqrt{c_{1}^{* 2}+4 m(1-\varepsilon-a)(1-\varepsilon)^{m+p-1}}-c_{1}^{*}\right) / 2 m\right) \xi} .
\end{aligned}
$$

By the arbitrariness of $\varepsilon>0$, (149)-(150) hold.

Theorem 20. Let $\varphi(\xi)$ be the increasing traveling wave solution of the problem (8)-(9) corresponding to the unique wave speed $c_{1}^{*}$.

(i) If $m>\min \{p, 1\}$, then $\xi_{a}>-\infty$.

(ii) If $m \leq \min \{p, 1\}$, then $\xi_{a}=-\infty$. More precisely,

(a) when $m+p>2$, if $m=1$, one has

$$
\varphi(\xi) \sim e^{c_{1}^{*} \xi}, \quad \text { as } \xi \longrightarrow-\infty
$$

and if $m<1$, one has

$$
\varphi(\xi) \sim\left(\frac{(m-1) c_{1}^{*}}{m} \xi\right)^{1 /(m-1)}, \quad \text { as } \xi \longrightarrow-\infty
$$

(b) when $m+p=2$, if $m=1$, one has

$$
\begin{gathered}
\varphi(\xi) \sim e^{\left(\left(\sqrt{c_{1}^{* 2}+4 a}+c_{1}^{*}\right) / 2\right) \xi}, \quad \text { as } \xi \longrightarrow-\infty, \\
\text { and if } m<1 \text {, one has } \\
\varphi(\xi) \sim\left(\frac{(m-1)\left(\sqrt{c_{1}^{* 2}+4 m a}+c_{1}^{*}\right)}{2 m} \xi\right)^{1 /(m-1)}, \\
\text { as } \xi \longrightarrow-\infty ;
\end{gathered}
$$

(c) when $1<m+p<2$, noticing that $m \leq$ $\min \{p, 1\}$, then $m \leq p$; if $m=p$, one has

$$
\varphi(\xi) \sim e^{(\sqrt{a} / m) \xi}, \quad \text { as } \xi \longrightarrow-\infty,
$$

and if $m<p$, one has

$$
\varphi(\xi) \sim\left(\frac{m-p}{2 m} \sqrt{\frac{2 m a}{m+p}} \xi\right)^{2 /(m-p)} \quad, \quad \text { as } \xi \longrightarrow-\infty
$$

Proof. For any $\varphi_{0} \in(0, a)$, we see that

$$
\xi(\varphi)=\xi\left(\varphi_{0}\right)-\int_{\varphi}^{\varphi_{0}} \frac{m s^{m-1}}{\psi(s)} d s .
$$

From the proof of Lemma 3, we see that when $\varphi>0$ is sufficiently small,

$$
\psi(\varphi)=A \varphi^{\gamma}+o\left(\varphi^{\gamma}\right)
$$

where $\gamma=1$ for $m+p \geq 2$, and $\gamma=(m+p) / 2$ for $1<m+p<$ 2. It is clear that $\xi(\varphi) \rightarrow-\infty$ as $\varphi \rightarrow 0^{+}$if $\gamma \geq m$, and $\xi(\varphi)$ is finite as $\varphi \rightarrow 0^{+}$if $\gamma<m$. That is, if $m>\min \{p, 1\}$, then $\xi_{a}>-\infty$; however, if $m \leq \min \{p, 1\}$, then $\xi_{a}=-\infty$. Furthermore, notice that as $\varphi \rightarrow 0^{+}$, when $m+p>2$,

$$
\psi(\varphi)=c_{1}^{*} \varphi+o(\varphi) .
$$

If $m=1$, we have

$$
\lim _{\varphi \rightarrow 0^{+}} \frac{\xi(\varphi)}{\ln \varphi}=\frac{1}{c_{1}^{*}}
$$

If $m<1$,

$$
\lim _{\varphi \rightarrow 0^{+}} \frac{\xi(\varphi)}{\varphi^{m-1}}=\frac{m}{(m-1) c_{1}^{*}} .
$$

When $m+p=2$,

$$
\psi(\varphi)=\frac{\sqrt{c_{1}^{* 2}+4 m a}+c}{2} \varphi+o(\varphi) .
$$

If $m=1$, we have

$$
\lim _{\varphi \rightarrow 0^{+}} \frac{\xi(\varphi)}{\ln \varphi}=\frac{\sqrt{c_{1}^{* 2}+4 a}-c_{1}^{*}}{2 a} .
$$

If $m<1$,

$$
\lim _{\varphi \rightarrow 0^{+}} \frac{\xi(\varphi)}{\varphi^{m-1}}=\frac{\sqrt{c_{1}^{* 2}+4 m a}-c_{1}^{*}}{2 a(m-1)} .
$$

When $1<m+p<2$,

$$
\psi(\varphi)=\sqrt{\frac{2 m a}{m+p}} \varphi^{(m+p) / 2}+o\left(\varphi^{(m+p) / 2}\right) .
$$


If $m=p$, we have

$$
\lim _{\varphi \rightarrow 0^{+}} \frac{\xi(\varphi)}{\ln \varphi}=\frac{m}{\sqrt{a}}
$$

If $m<p$,

$$
\lim _{\varphi \rightarrow 0^{+}} \frac{\xi(\varphi)}{\varphi^{(m-p) / 2}}=\frac{2 m}{m-p} \sqrt{\frac{m+p}{2 m a}} .
$$

By a simple calculation, (182)-(187) hold.

Theorem 21. Let $\varphi(\xi)$ be the decreasing traveling wave solution of the problem (10)-(11) corresponding to the unique wave speed $c_{2}^{*}$.

(i) If $0<q<1$, then $\tilde{\xi}_{a}>-\infty$.

(ii) If $q \geq 1$, then $\widetilde{\xi}_{a}=-\infty$. More precisely,

(a) when $q>1$, one has

$$
1-\varphi(\xi) \sim e^{\left(c_{2}^{*} / m\right) \xi}, \quad \text { as } \xi \longrightarrow-\infty ;
$$

(b) when $q=1$, one has

$$
1-\varphi(\xi) \sim e^{\left(\left(\sqrt{c_{2}^{* 2}+4 m(1-a)}+c_{2}^{*}\right) / 2 m\right) \xi}, \quad \text { as } \xi \longrightarrow-\infty .
$$

Proof. Let $\varphi_{0}$ approach 1 enough. Then, we have

$$
\xi\left(\varphi_{0}\right)-\xi(\varphi)=\int_{\varphi}^{\varphi_{0}} \frac{m s^{m-1}}{\psi(s)} d s .
$$

From the proof of Lemma 12, we see that

$$
\psi(\varphi) \sim A(1-\varphi)^{\gamma}
$$

as $\varphi \rightarrow 1^{-}$, where $\gamma=1$ for $q \geq 1$, and $\gamma=(q+1) / 2$ for $0<q<1$. It is clear that $\xi(\varphi) \rightarrow-\infty$ as $\varphi \rightarrow 1^{-}$if $\gamma \geq 1$, and $\xi(\varphi)$ is finite as $\varphi \rightarrow 1^{-}$if $\gamma<1$. That is, if $0<q<1$, then $\tilde{\xi}_{a}>-\infty$; however, if $q \geq 1$, then $\tilde{\xi}_{a}=-\infty$. Furthermore, notice that as $\varphi \rightarrow 1^{-}$, when $q>1$,

$$
\begin{gathered}
\psi(\varphi)=c_{2}^{*}(\varphi-1)+o(\varphi-1), \\
\lim _{\varphi \rightarrow 1^{-}} \frac{\xi(\varphi)}{\ln (1-\varphi)}=\frac{m}{c_{2}^{*}} .
\end{gathered}
$$

When $q=1$,

$$
\begin{gathered}
\psi(\varphi)=\frac{\sqrt{c_{2}^{* 2}+4 m(1-a)}+c_{2}^{*}}{2}(\varphi-1)+o(\varphi-1), \\
\lim _{\varphi \rightarrow 1^{-}} \frac{\xi(\varphi)}{\ln (1-\varphi)}=\frac{2 m}{\sqrt{c_{2}^{* 2}+4 m(1-a)}+c_{2}^{*}} .
\end{gathered}
$$

This yields (199)-(200).
Theorem 22. Let $\varphi(\xi)$ be the decreasing traveling wave solution of the problem (10)-(11) corresponding to the unique wave speed $c_{2}^{*}$.

(i) If $p<\min \{m, 1\}$, then $\widetilde{\xi}_{b}<+\infty$.

(ii) If $p \geq \min \{m, 1\}$, then $\tilde{\xi}_{b}=+\infty$. More precisely,

(a) when $m+p>2$, if $p>1$, one has

$$
\begin{gathered}
\varphi(\xi) \sim\left(\frac{a(p-1)}{c_{2}^{*}} \xi\right)^{1 /(1-p)}, \text { as } \xi \longrightarrow+\infty \\
\text { and if } p=1 \text {, one has } \\
\varphi(\xi) \sim e^{-\left(a / c_{2}^{*}\right) \xi}, \quad \text { as } \xi \longrightarrow+\infty
\end{gathered}
$$

(b) when $m+p=2$, if $p>1$, one has

$$
\varphi(\xi) \sim\left(\frac{(1-m)\left(\sqrt{c_{2}^{* 2}+4 m a}-c_{2}^{*}\right)}{2 m} \xi\right)^{1 /(m-1)}
$$

as $\xi \longrightarrow+\infty$,

and if $p=1$, one has

$$
\varphi(\xi) \sim e^{-\left(\left(\sqrt{c_{c}^{* 2}+4 a}-c_{2}^{*}\right) / 2\right) \xi}, \quad \text { as } \xi \longrightarrow+\infty
$$

(c) when $1<m+p<2$, noticing that $p \geq$ $\min \{m, 1\}$, then $p \geq m$; if $p>m$, one has

$$
\varphi(\xi) \sim\left(\frac{p-m}{2 m} \sqrt{\frac{2 m a}{m+p}} \xi\right)^{2 /(m-p)} \quad, \quad \text { as } \xi \longrightarrow+\infty
$$

and if $p=m$, one has

$$
\varphi(\xi) \sim e^{-(\sqrt{a} / m) \xi}, \quad \text { as } \xi \longrightarrow+\infty .
$$

Proof. (i) For $0<\varphi_{c_{2}^{*} \tau} \leq a / 2$, we have $0 \leq \varphi \leq \varphi_{c_{2}^{*} \tau} \leq a / 2$, and

$$
\begin{aligned}
\frac{1}{2} \frac{d \psi^{2}}{d \varphi}-c_{2}^{*} \psi & =-m \varphi^{m+p-1}(1-\varphi)^{q}\left(\varphi_{c_{2}^{*} \tau}-a\right) \\
& \geq \frac{m a}{2}\left(1-\frac{a}{2}\right)^{q} \varphi^{m+p-1} .
\end{aligned}
$$

Consider the following inequality problem:

$$
\begin{gathered}
\frac{1}{2} \frac{d u^{2}}{d \varphi}-c_{2}^{*} u \leq \frac{m a}{2}\left(1-\frac{a}{2}\right)^{q} \varphi^{m+p-1}, \quad \text { for } \varphi \geq 0, \\
u\left(0^{+}\right)=0, \\
u(\varphi)<0, \quad \text { for } \varphi>0 .
\end{gathered}
$$


It is easy to prove that $\psi(\varphi) \leq u(\varphi)$ when $\varphi_{c_{2}^{*} \tau} \leq a / 2$. Now, we construct a function $u$ satisfying (212). Let

$$
u=-B \varphi^{r} \text {. }
$$

Then, $u$ satisfies (212) if and only if

$$
B^{2} r \varphi^{2 r-m-p}+c_{2}^{*} B \varphi^{r-m-p+1} \leq \frac{m a}{2}\left(1-\frac{a}{2}\right)^{q} .
$$

Take

$$
r=\max \left\{\frac{m+p}{2}, m+p-1\right\}
$$

Then, (212) holds when $B$ is appropriately small. Thus,

$$
\psi(\varphi) \leq-B \varphi^{r}
$$

with $r=\max \{(m+p) / 2, m+p-1\}$ and $B$ appropriately small. Since $\varphi(\xi)$ is the solution of the problem (10)-(11), there exists $\varphi_{0}>0$ sufficiently small such that

$$
\begin{aligned}
\xi(\varphi)-\xi\left(\varphi_{0}\right) & =-\int_{\varphi}^{\varphi_{0}} \frac{m s^{m-1}}{\psi(s)} d s \\
& \leq \frac{m}{B} \int_{\varphi}^{\varphi_{0}} \frac{1}{s^{\theta-m+1}} d s
\end{aligned}
$$

When $p<\min \{m, 1\}, \xi(\varphi)<+\infty$ as $\varphi \rightarrow 0^{+}$. Thus, $\tilde{\xi}_{b}<$ $+\infty$.

(ii) From the proof of Theorem 14, we know that

$$
\begin{aligned}
\psi(\varphi) & \geq \tilde{\psi}^{*}(\varphi)=-\sqrt{2 m \int_{0}^{\varphi} s^{m+p-1}(1-s)^{q}(a-s) d s} \\
& \geq-\sqrt{\frac{2 m a}{m+p}} \varphi^{(m+p) / 2} .
\end{aligned}
$$

On the other hand, from the proof of Theorem 17, we also note that

$$
\psi(\varphi) \geq-\frac{m a}{c_{2}^{*}} \varphi^{m+p-1}
$$

By (218)-(219) and

$$
\xi(\varphi)-\xi\left(\varphi_{0}\right)=\int_{\varphi_{0}}^{\varphi} \frac{m s^{m-1}}{\psi(s)} d s,
$$

it is easy to see that

$$
\lim _{\varphi \rightarrow 0^{+}} \xi(\varphi)=+\infty
$$

if $p \geq \min \{m, 1\}$. That is,

$$
\tilde{\xi}_{b}=+\infty
$$

For any $0<\varepsilon<a$, when $\varphi$ approaches 0 enough, we have $\varphi_{c_{2}^{*} \tau}<\varepsilon$. Consider the following problem:

$$
\begin{gathered}
\frac{1}{2} \frac{d u^{2}}{d \varphi}-c_{2}^{*} u \leq m \varphi^{m+p-1}(1-\varphi)^{q}(a-\varepsilon), \quad \text { for } \varphi \geq 0, \\
u\left(0^{+}\right)=0, \\
u(\varphi)<0, \quad \text { for } \varphi>0 .
\end{gathered}
$$

It is easy to prove that $\psi(\varphi) \leq u(\varphi)$ when $\varphi>0$ with $\varphi_{c_{2}^{*} \tau}<\varepsilon$. In what follows, we construct a function $u$ satisfying (223). Let

$$
u=-B \varphi^{r}
$$

Noticing that $0<\varphi \leq \varphi_{c_{2}^{*} \tau}<\mathcal{E}$, then $u$ satisfies (223) if

$$
B^{2} r \varphi^{2 r-m-p}+c_{2}^{*} B \varphi^{r-m-p+1} \leq m(1-\varepsilon)^{q}(a-\varepsilon) .
$$

When $m+p>2$, take $r=m+p-1$. Then, (225) is ensured by the following inequalities:

$$
\begin{gathered}
c_{2}^{*} B \leq m(1-\varepsilon)^{q}(a-\varepsilon)\left(1-\varepsilon^{(m+p-2) / 2}\right), \\
(m+p-1) B^{2} q \varepsilon^{(m+p-2) / 2} \leq m(1-\varepsilon)^{q}(a-\varepsilon) .
\end{gathered}
$$

Take

$$
B=\frac{m(1-\varepsilon)^{q}(a-\varepsilon)\left(1-\varepsilon^{(m+p-2) / 2}\right)}{c_{2}^{*}} .
$$

Then, when $\varepsilon<\left(c_{2}^{* 2} / m(m+p-1)\right)^{2 /(m+p-2)}$, (225) holds. Recalling (219), we obtain

$$
\begin{aligned}
& -\frac{m a}{c_{2}^{*}} \varphi^{m+p-1} \\
& \leq \psi(\varphi) \\
& \quad \leq-\frac{m(1-\varepsilon)^{q}(a-\varepsilon)\left(1-\varepsilon^{(m+p-2) / 2}\right)}{c_{2}^{*}} \varphi^{m+p-1}
\end{aligned}
$$

when $\varphi_{c_{2}^{*} \tau}<\varepsilon$. When $p>1$, we have

$$
\begin{aligned}
& \frac{c_{2}^{*}}{(1-\varepsilon)^{q}(a-\varepsilon)\left(1-\varepsilon^{(m+p-2) / 2}\right)(p-1)} \\
& \quad \leq \lim _{\varphi \rightarrow 0^{+}} \xi(\varphi) \varphi^{p-1} \\
& \quad \leq \frac{c_{2}^{*}}{a(p-1)} ;
\end{aligned}
$$

that is,

$$
\begin{aligned}
& \left(\frac{(1-\varepsilon)^{q}(a-\varepsilon)\left(1-\varepsilon^{(m+p-2) / 2}\right)(p-1)}{c_{2}^{*}}\right)^{1 /(1-p)} \\
& \leq \lim _{\xi \rightarrow+\infty} \varphi(\xi) \xi^{1 /(p-1)} \leq\left(\frac{a(p-1)}{c_{2}^{*}}\right)^{1 /(1-p)} .
\end{aligned}
$$


When $p=1$, we have

$$
-\frac{c_{2}^{*}}{(1-\varepsilon)^{q}(a-\varepsilon)\left(1-\varepsilon^{(m+p-2) / 2}\right)} \leq \lim _{\varphi \rightarrow 0^{+}} \frac{\xi(\varphi)}{\ln \varphi} \leq-\frac{c_{2}^{*}}{a} ;
$$

that is,

$$
e^{-\left(a / c_{2}^{*}\right) \xi} \leq \varphi(\xi) \leq e^{-\left((1-\varepsilon)^{q}(a-\varepsilon)\left(1-\varepsilon^{(m+p-2) / 2}\right) / c_{2}^{*}\right) \xi} .
$$

By the arbitrariness of $\varepsilon>0$, (205)-(206) hold.

When $1<m+p<2$, take $r=(m+p) / 2$. Then, (225) holds if

$$
\begin{gathered}
\frac{m+p}{2} B^{2} \leq m(1-\varepsilon)^{q}(a-\varepsilon)\left(1-\varepsilon^{(2-m-p) / 4}\right), \\
c_{2}^{*} B \varepsilon^{(2-m-p) / 4} \leq m(1-\varepsilon)^{q}(a-\varepsilon) .
\end{gathered}
$$

Take

$$
B=\sqrt{\frac{2 m}{m+p}(1-\varepsilon)^{q}(a-\varepsilon)\left(1-\varepsilon^{(2-m-p) / 4}\right)} .
$$

Then, when

$$
\begin{aligned}
\varepsilon \leq \min & \left\{\frac{a}{2},\left(\frac{a}{2}\left(1-\frac{a}{2}\right)^{q}\right)^{2 /(2-m-p)}\right. \\
& \left.\times\left(\frac{m(m+p)}{2} c_{2}^{*-2}\right)^{2 /(2-m-p)}\right\},
\end{aligned}
$$

(225) holds. Combining with (218), we obtain

$$
\begin{aligned}
& -\sqrt{\frac{2 m a}{m+p}} \varphi^{(m+p) / 2} \leq \psi(\varphi) \\
& \leq-\sqrt{\frac{2 m}{m+p}(1-\varepsilon)^{q}(a-\varepsilon)\left(1-\varepsilon^{(2-m-p) / 4}\right)} \varphi^{(m+p) / 2}
\end{aligned}
$$

when $\varphi_{c_{2}^{*} \tau}<\varepsilon$. When $p>m$, a simple calculation yields

$$
\begin{gathered}
\left(\frac{p-m}{2 m} \sqrt{\frac{2 m(1-\varepsilon)^{q}(a-\varepsilon)\left(1-\varepsilon^{(2-m-p) / 4}\right)}{m+p}}\right)^{2 /(m-p)} \\
\leq \lim _{\xi \rightarrow+\infty} \varphi(\xi) \xi^{2 /(p-m)} \leq\left(\frac{p-m}{2 m} \sqrt{\frac{2 m a}{m+p}}\right)^{2 /(m-p)}
\end{gathered}
$$

When $p=m$,

$$
e^{-(\sqrt{a} / m) \xi} \leq \varphi(\xi) \leq e^{-\left(\sqrt{(1-\varepsilon)^{q}(a-\varepsilon)\left(1-\varepsilon^{(1-m) / 2}\right) / m}\right) \xi} .
$$

By the arbitrariness of $\varepsilon>0$, (209)-(210) hold.

When $m+p=2$, take

$$
r=1, \quad B=\frac{\sqrt{c_{2}^{* 2}+4 m(1-\varepsilon)^{q}(a-\varepsilon)}-c_{2}^{*}}{2} .
$$

Then, (225) holds. On the other hand, consider the following problem:

$$
\begin{gathered}
\frac{1}{2} \frac{d v^{2}}{d \varphi}-c_{2}^{*} v \geq \operatorname{ma\varphi }(1-\varphi)^{q}, \quad \text { for } \varphi \geq 0, \\
v\left(0^{+}\right)=0, \\
v(\varphi)<0, \quad \text { for } \varphi>0 .
\end{gathered}
$$

We can see that $\psi(\varphi) \geq v(\varphi)$. Let

$$
v=-\frac{\sqrt{c_{2}^{* 2}+4 m a}-c_{2}^{*}}{2} \varphi .
$$

Then, $v$ satisfies (240). Thus, we conclude that

$$
\begin{aligned}
& -\frac{\sqrt{c_{2}^{* 2}+4 m a}-c_{2}^{*}}{2} \varphi \\
& \leq \psi(\varphi) \\
& \quad \leq-\frac{\sqrt{c_{2}^{* 2}+4 m(1-\varepsilon)^{q}(a-\varepsilon)}-c_{2}^{*}}{2} \varphi .
\end{aligned}
$$

When $m<1$, that is, $p>1$,

$$
\begin{aligned}
& \left(\frac{(1-m)\left(\sqrt{c_{2}^{* 2}+4 m(1-\varepsilon)^{q}(a-\varepsilon)}-c_{2}^{*}\right)}{2 m}\right)^{1 /(m-1)} \\
& \leq \lim _{\xi \rightarrow+\infty} \varphi(\xi) \xi^{1 /(1-m)} \\
& \leq\left(\frac{(1-m)\left(\sqrt{c_{2}^{* 2}+4 m a}-c_{2}^{*}\right)}{2 m}\right)^{1 /(m-1)}
\end{aligned}
$$

When $m=1$, that is, $p=1$,

$$
\begin{aligned}
e^{-\left(\left(\sqrt{c_{2}^{* 2}+4 a}-c_{2}^{*}\right) / 2\right) \xi} & \leq \varphi(\xi) \\
& \leq e^{-\left(\left(\sqrt{c_{2}^{* 2}+4(1-\varepsilon)^{q}(a-\varepsilon)}-c_{2}^{*}\right) / 2\right) \xi}
\end{aligned}
$$

By the arbitrariness of $\varepsilon>0$, (207)-(208) hold.

\section{Discussion}

When $p=q=1$, the outcome in our work is reduced to the results obtained in [23]. Comparing with [23], our definition of sharp-type traveling wave fronts and smooth-type traveling wave fronts is more precise. In the proof of Lemma 3, for the case $m+p=2$, we constructed two sequences to get the asymptotic expression of $\psi(\varphi)$ for $\varphi>0$ sufficiently small. This technique is not used in [23]. Moreover, the proof of Theorem 17 is more concise than the proof of Proposition 2.7 in [23]. 


\section{Acknowledgments}

The authors are grateful to the anonymous reviewers for the careful reading and helpful suggestions which led to an improvement of their original paper. This research is supported by the Fundamental Research Funds for the Central Universities (no. 2012ZM0057), the National Science Foundation of Guangdong Province (no. S2012040007959), and the National Natural Science Foundation of China (no. 11171115).

\section{References}

[1] A. L. Hodgkin and A. F. Huxley, "A quantitative description of membrane current and its application to conduction and excitation in nerve," The Journal of Physiology, vol. 117, no. 4, pp. 500-544, 1952.

[2] S. A. Gourley, "Travelling fronts in the diffusive Nicholson's blowflies equation with distributed delays," Mathematical and Computer Modelling, vol. 32, no. 7-8, pp. 843-853, 2000.

[3] G. Lin and W. T. Li, "Bistable wavefronts in a diffusive and competitive Lotka-Volterra type system with nonlocal delays," Journal of Differential Equations, vol. 244, no. 3, pp. 487-513, 2008.

[4] W. Yan and R. Liu, "Existence and critical speed of traveling wave fronts in a modified vector disease model with distributed delay," Journal of Dynamical and Control Systems, vol. 18, no. 3, pp. 355-378, 2012.

[5] Y. Zhu, Y. Cai, S. Yan, and W. Wang, "Dynamical analysis of a delayed reaction-diffusion predator-prey system," Abstract and Applied Analysis, vol. 2012, Article ID 323186, 23 pages, 2012.

[6] B. I. Camara and H. Mokrani, "Analysis of wave solutions of an adhenovirus-tumor cell system," Abstract and Applied Analysis, vol. 2012, Article ID 590326, 13 pages, 2012.

[7] N. Min, H. Zhang, and Z. Qu, "Dynamic properties of a forest fire model," Abstract and Applied Analysis, vol. 2012, Article ID 948480, 13 pages, 2012.

[8] D. G. Aronson and H. F. Weinberger, "Nonlinear diffusion in population genetics, combustion, and nerve pulse propagation," in Partial Differential Equations and Related Topics, pp. 5-49, Springer, Berlin, Germany, 1975.

[9] P. C. Fife and J. B. McLeod, "The approach of solutions of nonlinear diffusion equations to travelling front solutions," Archive for Rational Mechanics and Analysis, vol. 65, no. 4, pp. 335-361, 1977.

[10] D. G. Aronson and H. F. Weinberger, "Multidimensional nonlinear diffusion arising in population genetics," Advances in Mathematics, vol. 30, no. 1, pp. 33-76, 1978.

[11] D. G. Aronson, "Density-dependent interaction-diffusion systems," in Dynamics and Modelling of Reactive Systems, vol. 44, pp. 161-176, Academic Press, New York, NY, USA, 1980.

[12] Y. Hosono, "Traveling wave solutions for some density dependent diffusion equations," Japan Journal of Applied Mathematics, vol. 3, no. 1, pp. 163-196, 1986.

[13] F. Sánchez-Garduño and P. K. Maini, "Travelling wave phenomena in non-linear diffusion degenerate Nagumo equations," Journal of Mathematical Biology, vol. 35, no. 6, pp. 713-728, 1997.

[14] H. Engler, "Relations between travelling wave solutions of quasilinear parabolic equations," Proceedings of the American Mathematical Society, vol. 93, no. 2, pp. 297-302, 1985.
[15] A. de Pablo and J. L. Vazquez, "Travelling waves and finite propagation in a reaction-diffusion equation," Journal of Differential Equations, vol. 93, no. 1, pp. 19-61, 1991.

[16] F. Sanchez-Garduno and P. K. Maini, "Existence and uniqueness of a sharp travelling wave in degenerate non-linear diffusion Fisher-KPP equations," Journal of Mathematical Biology, vol. 33, no. 2, pp. 163-192, 1994.

[17] F. Sanchez-Garduno and P. K. Maini, "Travelling wave phenomena in some degenerate reaction-diffusion equations," Journal of Differential Equations, vol. 117, no. 2, pp. 281-319, 1995.

[18] F. Sanchez-Garduno, P. K. Maini, and M. E. Kappos, "A shooting argument approach to a sharp-type solution for nonlinear degenerate Fisher-KPP equations," IMA Journal of Applied Mathematics, vol. 57, no. 3, pp. 211-221, 1996.

[19] J. A. Sherratt and B. P. Marchant, "Nonsharp travelling wave fronts in the Fisher equation with degenerate nonlinear diffusion," Applied Mathematics Letters, vol. 9, no. 5, pp. 33-38, 1996.

[20] A. de Pablo and A. Sanchez, "Global travelling waves in reaction-convection-diffusion equations," Journal of Differential Equations, vol. 165, no. 2, pp. 377-413, 2000.

[21] L. Malaguti and C. Marcelli, "Sharp profiles in degenerate and doubly degenerate Fisher-KPP equations," Journal of Differential Equations, vol. 195, no. 2, pp. 471-496, 2003.

[22] B. H. Gilding and R. Kersner, "A Fisher/KPP-type equation with density-dependent diffusion and convection: travelling-wave solutions," Journal of Physics A, vol. 38, no. 15, pp. 3367-3379, 2005.

[23] C. Jin, J. Yin, and S. Zheng, "Traveling waves for a time delayed Newtonian filtration equation," Journal of Differential Equations, vol. 254, no. 1, pp. 1-29, 2013.

[24] C. Jin and J. Yin, "Traveling wavefronts for a time delayed nonNewtonian filtration equation," Physica D, vol. 241, no. 21, pp. 1789-1803, 2012. 


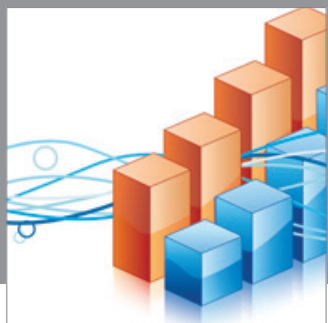

Advances in

Operations Research

mansans

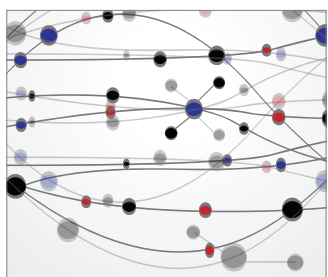

The Scientific World Journal
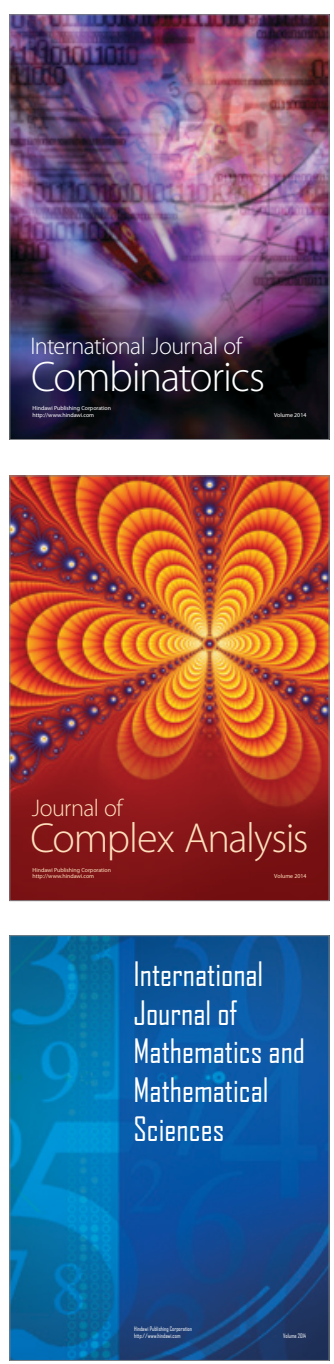
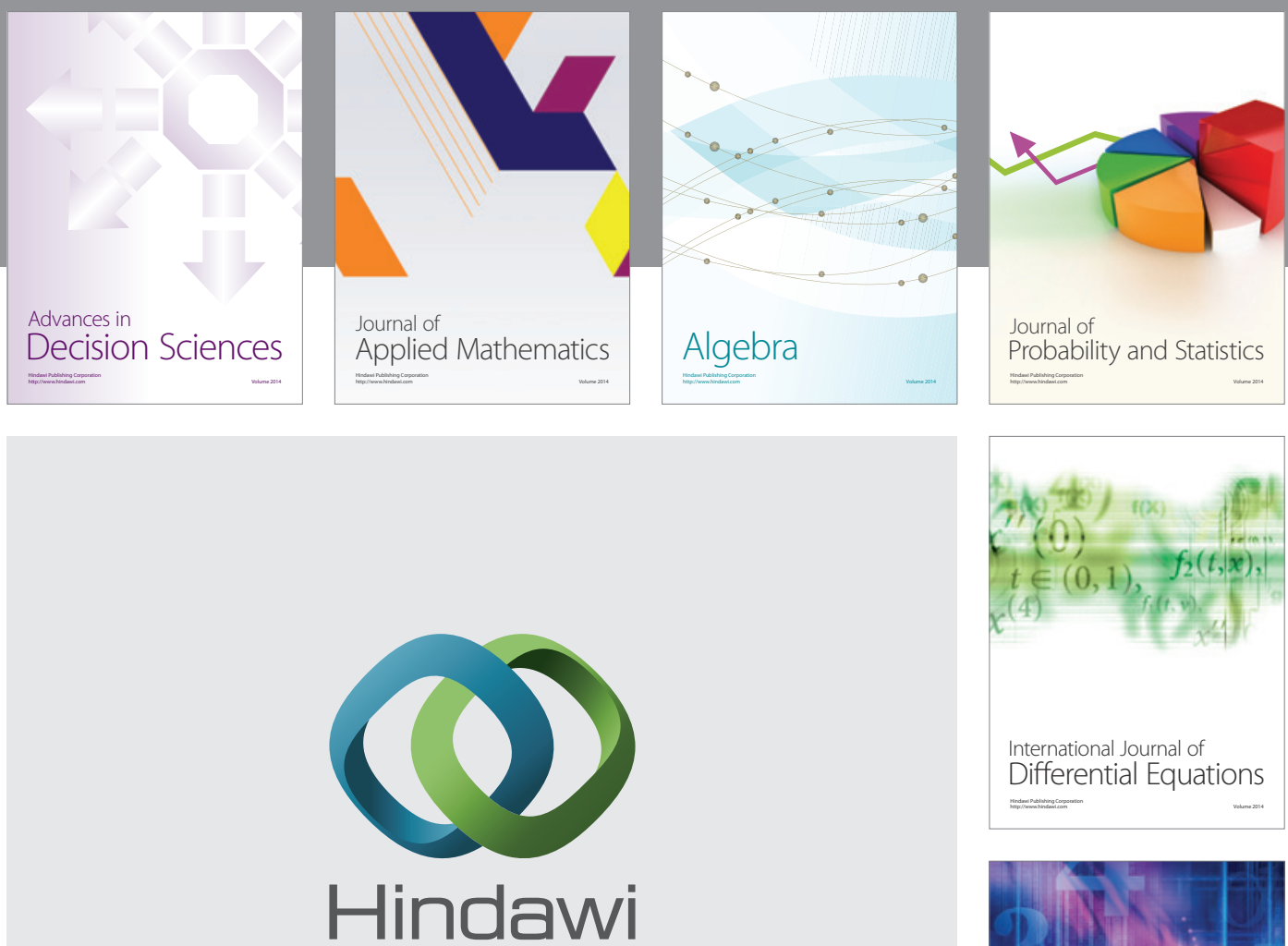

Submit your manuscripts at http://www.hindawi.com
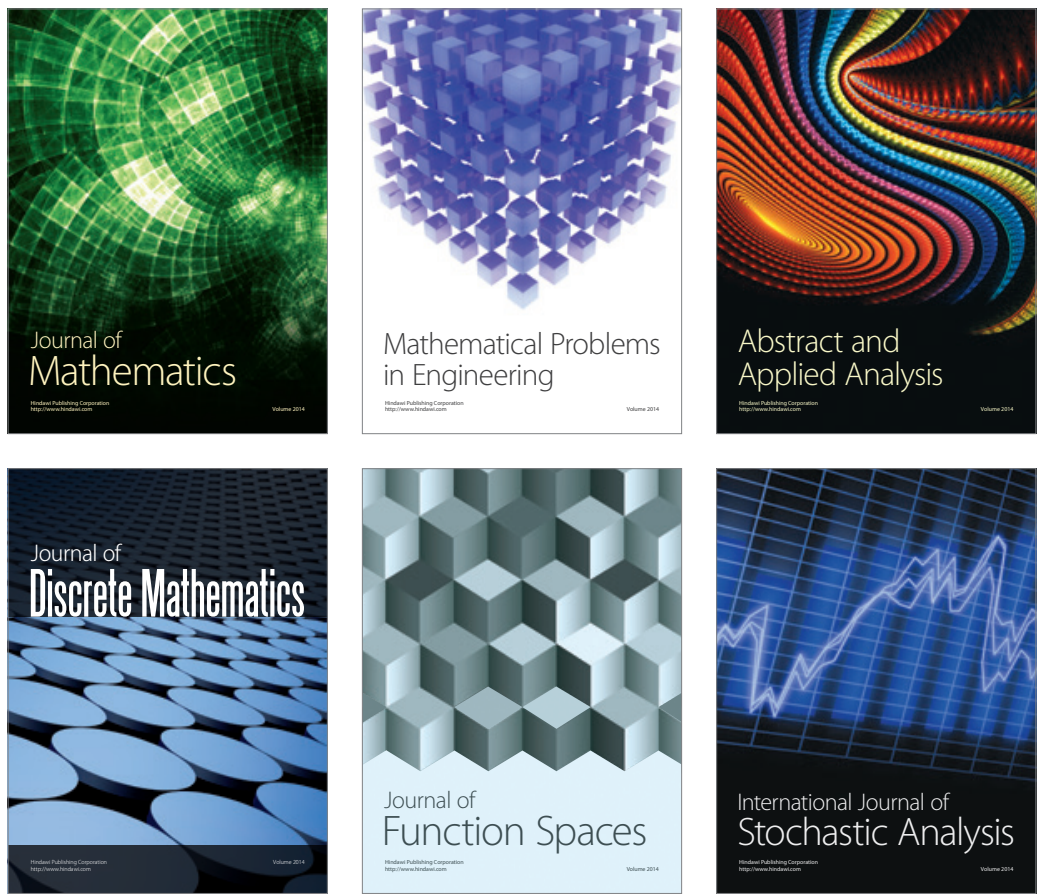

Journal of

Function Spaces

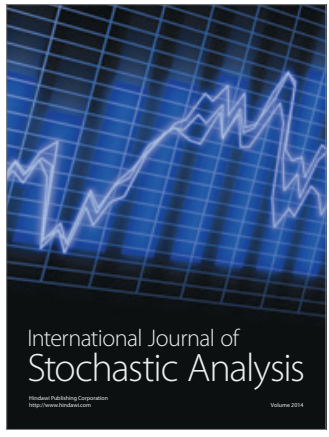

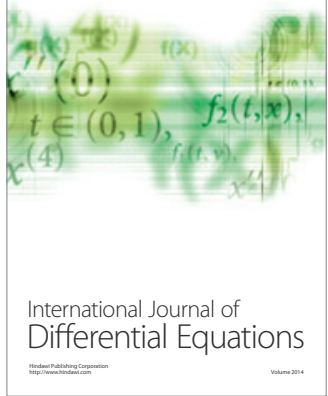
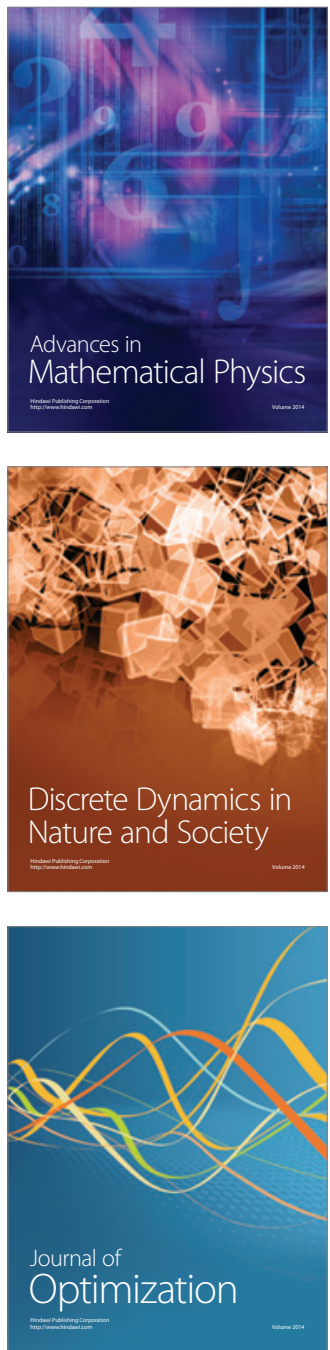